\title{
Dynamical modeling of the H3K27 epigenetic landscape in mouse embryonic stem cells
}

\author{
Kapil Newar ${ }^{1}$, Eric Fanchon ${ }^{1}$ and Daniel Jost ${ }^{1,2,{ }^{*}}$ \\ ${ }^{1}$ Univ Grenoble Alpes, CNRS, Grenoble INP, TIMC-IMAG, Grenoble, France \\ 2 Université de Lyon, ENS de Lyon, Univ Claude Bernard, CNRS, Laboratoire de Biologie et \\ Modélisation de la Cellule, Lyon, France \\ *To whom correspondence should be addressed: daniel.jost@ens-lyon.fr
}

\begin{abstract}
The Polycomb system via the methylation of the lysine 27 of histone H3 (H3K27) plays central roles in the silencing of many lineage-specific genes during development. Recent experimental evidence suggested that the recruitment of histone modifying enzymes like the Polycomb repressive complex $2(P R C 2)$ at specific sites and their spreading capacities from these sites are key to the establishment and maintenance of a proper epigenomic landscape around Polycombtarget genes. Here, to test whether such mechanisms, as a minimal set of qualitative rules, are quantitatively compatible with data, we developed a mathematical model that can predict the locus-specific distributions of H3K27 modifications based on previous biochemical knowledge. Within the biological context of mouse embryonic stem cells, our model showed quantitative agreement with experimental profiles of H3K27 acetylation and methylation around Polycombtarget genes in wild-type and mutants. In particular, we demonstrated the key role of the readerwriter module of PRC2 and of the competition between the binding of activating and repressing enzymes in shaping the H3K27 landscape around transcriptional start sites. The predicted dynamics of establishment and maintenance of the repressive trimethylated H3K27 state suggest a slow accumulation, in perfect agreement with experiments. Our approach represents a first step towards a quantitative description of PcG regulation in various cellular contexts and provides a generic framework to better characterize epigenetic regulation in normal or disease situations.
\end{abstract}




\section{Introduction}

Cells sharing the same genetic information may have very different functions and phenotypes. The regulation of gene expression is central to control such cellular identity. In eukaryotes, a key layer of regulation lies in the modulation of the accessibility and recruitment of the molecules driving transcription to chromatin. In particular, biochemical modifications of DNA and of histone tails, the so-called epigenetic or epigenomic marks, are believed to be essential in controlling such modulation (1). Each cell type is characterized by a distinct sequence of epigenetic marks along the genome with specific modifications associated with active or silent regions (2). Such epigenetic information should be robust and maintained across DNA replication and cell divisions but may also need to be plastic and modified during development or to adapt to environmental cues (3). A key question at the heart of epigenetics is thus to characterize the generic principles and mechanisms regulating the establishment, maintenance and conversion of the epigenomic marks.

Experimental studies suggested along the years that the regulation of these marks follows similar rules (4-6): chromatin regulators like histone modifying enzymes (HMEs) are recruited at specific DNA sequences leading to the nucleation of an epigenetic signal that subsequently spread to form more or less extended domains along the genome. In particular, the spreading process was found to be driven by a variety of 'reader-writer' enzymes that can 'read' a given chromatin modification at a given locus and 'write' or 'remove' the same or another mark at other genomic positions $(1,7)$.

To formalize such rules, several mathematical models investigating the generic regulation of histone marks have been developed (8-17). In their simplest form, these models consider that the local chromatin state can switch between active and repressive marks $(18,19)$. They suggested that the reader-writer-eraser capacity of HMEs may generate positive feedback loops and cooperative effects in the system that are essential to provide stability to the local epigenetic state. Applications of such formalism, contextualized to specific marks at specific loci, have shown that it is fully consistent with many experimental observations (13,14,17,20-24). However, quantitative comparisons with experiments are still rare in particular on how epigenetic marks organize around the nucleation sites, which may bring crucial information on the spreading and maintenance mechanisms $(15,25)$. In this work, we aim to provide a modeling framework able to quantitatively describe the genomic profiles of epigenetic marks in the context of the Polycomb system in mouse embryonic stem cells (mESCs).

The Polycomb regulatory system is found in many higher eukaryotes and has been shown to play a critical role during development in the silencing of lineage-specific genes (26). It involves the methylation of the lysine 27 of histone $\mathrm{H} 3$ mainly via the coordinated action of two Polycombgroup (PcG) complexes, PRC1 and PRC2, tri-methylation of H3K27 (H3K27me3) being associated with gene repression.

mESCs have been for years a model system to investigate the Polycomb system in mammals (27), as it is involved in the maintenance of the pluripotency of these cells (28). Recently, many experimental studies in mESCs have measured quantitatively the patterns of H3K27 modifications 
along the genome $(6,25,29-33)$. Genes targeted by PcG proteins, the so-called PcG-target genes, are characterized by high H3K27me3 levels around their transcriptional start sites (TSS), low levels of H3K27me1 and me2 and the quasi-absence of H3K27ac (Fig.1B). Active genes are enriched with H3K27me1 and H3K27ac marks. Bivalent genes, associated with lowly expressed or poised regions and characterized by the co-occurrence of active (H3K4me3) and repressive marks (34), exhibit intermediate levels of $\mathrm{H} 3 \mathrm{~K} 27 \mathrm{me} 2$ and me3. The intergenic regions are dominated by H3K27me2 representing more than $50 \%$ of all H3K27 modifications (6).

Perturbations of this H3K27 landscape when altering PRC1/2 have allowed to shed light on the functions of their molecular constituents in the establishment and maintenance of the epigenetic signal (Fig.1A) (6,29,30,35-37). Briefly, the recruitment of a non-canonical PRC1 variant at CpG islands (CGIs) mediates locally the mono-ubiquitination of H2AK119 (35-40). This localized signal in turn recruits PRC2 through the interactions of cofactors like JARID2 (6,41-43). Around its core subunit Suz12, PRC2 contains the EZH1 or EZH2 catalytic subunit, both capable of methylating $\mathrm{H} 3 \mathrm{~K} 27$ and of nucleating the epigenetic signal. PRC2 includes also a 'reader' subunit, EED, that allosterically boosts the activity of the 'writer' EZH2 in presence of H3K27me3 residues (44) and allows the long-range spreading of the signal around the nucleation site (6). Canonical PRC1 may then bind to $\mathrm{H} 3 \mathrm{~K} 27$ me3-tagged regions leading to the local compaction of chromatin and repression of gene expression $(35-37,45)$. The silencing action of PcG proteins is antagonized by Trithorax-group proteins like MLL2 that recruit demethylases like UTX/JMJD3 and acetyltransferases like p300/CBP (46) mediating, respectively, the removal of the methyl groups from methylated $\mathrm{H} 3 \mathrm{~K} 27$ residues (47) or the addition of an acetyl group to unmarked H3K27 residues $(48,49)$, which is crucial for transcriptional activation.

All this suggests that the recruitment of HMEs at specific sites and their local and long-range spreading activities from these sites are designing the epigenetic H3K27 landscape in mESCs. While these different mechanisms were discovered and characterized in a heterogeneous set of in vivo and in vitro experimental assays, it is unclear whether, all together, they provide a complete set of processes that can quantitatively describe in vivo experimental epigenetic profiles.

Here, to address this question, we turn this experimentally-derived knowledge into a quantitative mechanistic model. Building on previous generic mathematical models of epigenetic regulation (see above), we contextualize our framework to precisely account for the occupancy of key HMEs and for the major processes described above. Using this integrated model, we investigate how the 'spreading' tug-of-war between the repressive (H3K27me3) and active (H3K27ac) marks is tied by the properties and locations of HMEs. In particular, we show that the model predictions are in quantitative agreement with Chip-Seq H3K27 profiles measured around PcG-target, active or bivalent genes in wild-type (WT) and perturbed conditions and with Stable Isotope Labeling by Amino-acids Cell culture (SILAC) experiments monitoring the maintenance and spreading dynamics of H3K27 methylation. We demonstrate the central role of the reader-writer module of PRC2 and of the competition between activating and repressing mechanisms in shaping the valency of H3K27 methylation, ie the relative levels of mono-, bi- and trimethylation around TSS. Finally, we conclude and discuss the perspectives and limitations of our approach. 


\section{Results}

\section{A dynamical model for the regulation of H3K27 epigenetic modifications in $\mathrm{mESC}$}

Based on previous experimental findings, we consider that the de novo establishment and maintenance of H3K27 modifications in mESC is mainly carried out by the stable recruitment of HMEs to their cognate DNA binding sites and that the patterns of epigenetic marks around genes result from a complex network of local and long-range spreading or erasing mechanisms mediated by these HMEs (6) (Fig.1A).
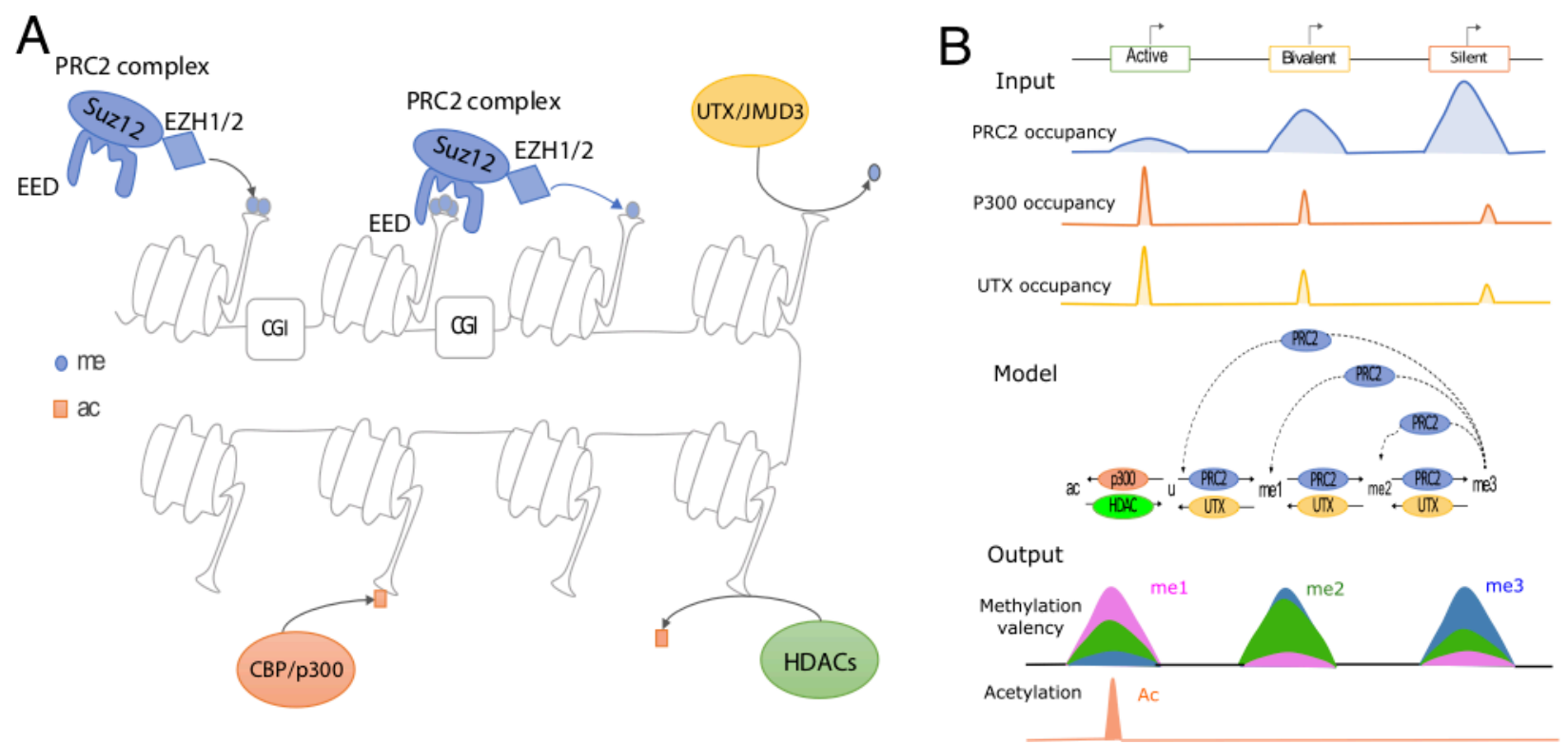

Figure 1. Model for the regulation of H3K27 modifications in mESCs. (A) Scheme of the different histone modifying enzymes (HMEs) involved in the regulation of H3K27 modifications. PRC2 complexes recruited to CpG densed regions contain: one of the two 'writers' (the methyltransferases Ezh1 and Ezh2) that methylates H3K27 and a 'reader' (EED) that binds to H3K27me3 and may allosterically activate EZH2. CBP/p300 acetylates the H3K27 residue, UTX/JMJD3 and HDACS remove the methyl and acetyl groups from H3K27, respectively. (B) Summary of the model. (Center) Multi-state dynamics of the H3K27 modifications: unmodified histones $(u)$ are methylated to me1, me2 and me3 or acetylated to ac by the action of PRC2 (local and long-range) and p300 (local), respectively. Demethylation is conditional to the local UTX occupancy while deacetylation by HDACs is considered uniform. The long-range spreading of methylation mediated by PRC2-H3K27me3 allosteric activation is shown as dashed lines. Histone turnover and DNA replication are not shown here for clarity. The model takes HME profiles as inputs (top) and makes predictions on the probabilities to find a given mark at a given position (bottom). (Top, Bottom) Graphical representations of the average occupancies of HMEs (top) and marks (bottom) expected around silent (PcG-target), active and bivalent genes. Methylation valency is defined as the relative ordering of H3K27me levels in the region. 
To test this hypothesis quantitatively, we simulated the stochastic dynamics of H3K27 modifications in a 20kbp-region around the TSS of a gene. This region, made of 100 nucleosomes, is modelled as an array of 200 histones where we assumed that each nucleosome ( 200bp) is made of two consecutive independent histones (each covering $\sim 100 \mathrm{bp}$ ). The H3K27 status of each histone can fluctuate among five states (Fig. 1B): unmodified (u), acetylated (ac), single-methylated (me1), double-methylated (me2) and tri-methylated (me3). Our model assumes that the dynamics of individual histones is mainly driven by the sequential addition or removal of acetyl and methyl groups by HMEs and by histone turnover (14,50-52). Below, we describe the three main features of this model (Fig. 1B). Their mathematical translation into reaction rates that control the stochastic transitions between histone states can be found in the Materials and Methods section.

\section{Addition and removal of the methyl groups by PRC2 and UTX}

Methylation of H3K27 is catalyzed by the PRC2 complex (53). In mammals, PRC2 is predominantly recruited at CpG islands (CGIs) by several cofactors including JARID2 $(6,41)$ or vPRC1-mediated H2AK119 mono-ubiquitination (35-40). Its methyltransferase activity is carried out by the subunits EZH1 or EZH2 (30). While the EZH1 activity remains largely local, interactions between the PRC2 subunit EED and H3K27me3-marked histones at the core recruitment region may allosterically boost the EZH2 activity which is then allowed, via a reader-writer mechanism, to spread methylation at long-range, outside the PRC2 cognate binding sites $(6,29,44)$. To account for this dual activity, we assumed that the methylation propensities at a given histone position are composed (i) by a local, nucleation term (of rate $k_{m e_{x}}$ with $x \in\{1,2,3\}$ ) proportional to the PRC2 occupancy at this position; and (ii) by a long-range term (of rate $\epsilon_{m e_{x}}$ ) accounting for the spreading capacity in 3D of distant PRC2 complexes bound to H3K27me3 histones at other positions that may spatially contact the locus by DNA looping (6). As $k_{m e_{x}}$ and $\epsilon_{m e_{x}}$ rates reflect the catalytic activity of the same complex (PRC2), we further considered that the ratios $\epsilon_{m e_{x}} / k_{m e_{x}}$ $(x \in\{1,2,3\})$, which characterizes the fold-change in effective activity of the allosterically-boosted, long-range spreading vs local nucleation, is state-independent, ie $\epsilon_{m e_{x}} / k_{m e_{x}}=R$ for all $x$ (see Materials and Methods).

H3K27 methyl groups can be actively removed by the demethylase UTX with no evidence suggesting that UTX "spreads" its activity at long-range (47). Therefore, we modeled the demethylation propensities as being simply proportional to the local UTX density with a rate $\gamma_{m e}$ that, to simplify, we assumed to be independent of the methylation status.

\section{Addition and removal of H3K27 acetylation}

At the promoters of active genes, acetylation of $\mathrm{H} 3 \mathrm{~K} 27$ is mediated by several acetyltransferases such as p300 or CBP recruited by transcription factors $(46,48,49)$. For p300-mediated acetylation, there is evidence suggesting that the bromodomain of p300 may trigger a reader-writer spreading process of acetylation (54), similar to the EZH2-mediated methylation. Such a mechanism would imply a long-range spreading of $\mathrm{H} 3 \mathrm{~K} 27 \mathrm{ac}$ around the p300 binding sites. However, after analyzing H3K27ac and p300 ChipSeq data around promoters, we found that p300 peaks are actually even slightly wider than the acetylation peaks (Figure 2B-C). Furthermore, while the inhibition of 
bromodomain enzymatic activity of p300 results in major loss of acetylation at enhancers, it only leads to minor changes at promoters (46). Since we aimed to describe acetylation at promoters, we neglected the bromodomain interplay and we simply assumed that the acetylation propensity is proportional to the $\mathrm{p} 300$ occupancy with a rate $k_{a c}$.

In general, deacetylation kinetics is fast and the half-lives of acetylated histone residues have been measured at many sites (55). Therefore, we modeled the action of histone deacetylases (HDACs) as a uniform rate of 0.6 event per hour (56) acting on H3K27ac histones.

Table 1: Parameters of the epigenetic model.

\begin{tabular}{|c|c|c|}
\hline Parameter & Description & Best value \\
\hline$k_{m e_{1}}$ & PRC2-mediated methylation rate $\left(u \rightarrow m e_{1}\right)$ & $3 \times k_{m e_{3}}$ \\
\hline$k_{m e_{2}}$ & PRC2-mediated methylation rate $\left(m e_{1} \rightarrow m e_{2}\right)$ & $3 \times k_{m e_{3}}$ \\
\hline$k_{m e_{3}}$ & PRC2-mediated methylation rate $\left(m e_{2} \rightarrow m e_{3}\right)$ & $0.81 \pm 0.1 h^{-1}$ \\
\hline$R$ & spreading-vs-nucleation ratio $\left(\epsilon_{m e_{x}} / k_{m e_{x}}\right)$ & $0.85 \pm 0.01$ \\
\hline$\epsilon_{m e_{1}}$ & $\mathrm{EZH} 2$ allosteric spreading rate $\left(u \rightarrow m e_{1}\right)$ & $R \times k_{m e_{1}}$ \\
\hline$\epsilon_{m e_{2}}$ & $\mathrm{EZH} 2$ allosteric spreading rate $\left(m e_{1} \rightarrow m e_{2}\right)$ & $R \times k_{m e_{2}}$ \\
\hline$\epsilon_{m e_{3}}$ & EZH2 allosteric spreading rate $\left(m e_{2} \rightarrow m e_{3}\right)$ & $R \times k_{m e_{3}}$ \\
\hline$\gamma_{m e}$ & UTX-mediated demethylation rate & $1.5 \pm 0.05 h^{-1}$ \\
\hline$k_{a c}$ & P300-mediated acetylation rate & $1.03 h^{-1}$ \\
\hline$\gamma_{a c}$ & deacetylation rate & $0.6 h^{-1}$ taken from $(64)$ \\
\hline$\gamma_{t u r n}$ & histone turnover rate & $0.03 h^{-1}$ taken from (58) \\
\hline$T$ & cell cycle length & $13.5 h$ taken from (63) \\
\hline
\end{tabular}

\section{Histone turnover and DNA replication}

In addition to the previous reactions that involve specific enzymes, the local state may be affected by histone turnover $(57,58)$. We assumed that this process leads to the replacement of the current histone state by a 'naive', unmodified (u) histone with a rate of 0.03 event per hour as measured consistently by two different studies $(58,59)$.

DNA replication is also a major perturbative event for the epigenome as the 'mother' epigenetic information is diluted among the two sister chromatids (60). Since mother histones are symmetrically redistributed $(61,62)$, we modeled replication as specific periodic events, occuring 
every 13.5 hour (the median cell cycle length in mESC (63)), where half of the histone states are randomly lost and replaced by a 'u' state.

All together, these three main features drive the dynamic transitions between the different states of H3K27. This epigenetic model takes as inputs the binding profiles of HMEs like PRC2, p300 or UTX (Fig.1B and S5) and, for a given set of (de)methylation and (de)acetylation rates (Table 1), makes predictions about the corresponding profiles of $\mathrm{H} 3 \mathrm{~K} 27 \mathrm{ac} / \mathrm{u} / \mathrm{me} 1 / \mathrm{me} 2 / \mathrm{me} 3$ modifications based on the simulations of many single-cell stochastic trajectories of the epigenome (see Materials and Methods).

\section{The model recapitulates the epigenetic landscape of polycomb- target genes under various conditions}

We first asked if our working hypotheses and the corresponding mathematical model are consistent with the average epigenetic landscape observed around PcG-target genes in mESCs grown in 2i medium (25) for which lots of data are available (see Table 2). For that, we designed a multi-step inference strategy (Figure 2) in order to fix, from available experimental data, the remaining-free parameters of the model, namely the methylation nucleation rates $\left(k_{m e 1 / m e 2 / m e 3}\right)$, the methylation spreading rates $\left(\epsilon_{m e 1 / m e 2 / m e 3}\right)$, the demethylation $\left(\gamma_{m e}\right)$ and acetylation $\left(k_{a c}\right)$ rates. In particular, we exploited data from wild-type and from perturbation experiments $(29,32)$ where the activities of some HMEs have been modified.

\section{Latent acetylation of Polycomb domains in $\mathrm{EZH1/2}$ double knockout}

In wild-type mESCs, the acetylated H3K27 histones sites are mostly spotted at the enhancers and promoters of active genes, overlapping with the genomic occupancy of both UTX and p300 $(49,57)$. Although p300 is present at the promoters of PcG-target genes (Fig.2B), there is almost zero H3K27 acetylation (orange circles in Fig.2I). However, on knocking out both methyltransferases EZH1 and EZH2 (29), PcG genes become significantly acetylated (grey circles in Fig. 2C) and a deregulation of gene expression is observed (29). In this DKO situation where $\mathrm{H} 3 \mathrm{~K} 27$ methylation is absent, the epigenetic model reduces to a simple two-state model between $u$ and $a c$ states (Fig. 2A). This allowed us to infer the acetylation rate $k_{a c}=1.03 h^{-1}$ based on the p300 average occupancy around PcG promoters (Fig.2B) by fitting the corresponding average $\mathrm{H} 3 \mathrm{~K} 27 \mathrm{ac}$ profiles (orange line in Fig.2C) (see Materials and Methods). Interestingly, our estimation of $k_{a c}$ is consistent with acetylation rates measured in human embryonic kidney cells for various residues after HDAC inhibition (65). Since $k_{a c}$ is of the same order than the HDACmediated deacetylation rate $\left(\sim 0.6 h^{-1}\right)$, it also suggests that acetylation levels result from a fast exchange dynamics of acetyl groups. 

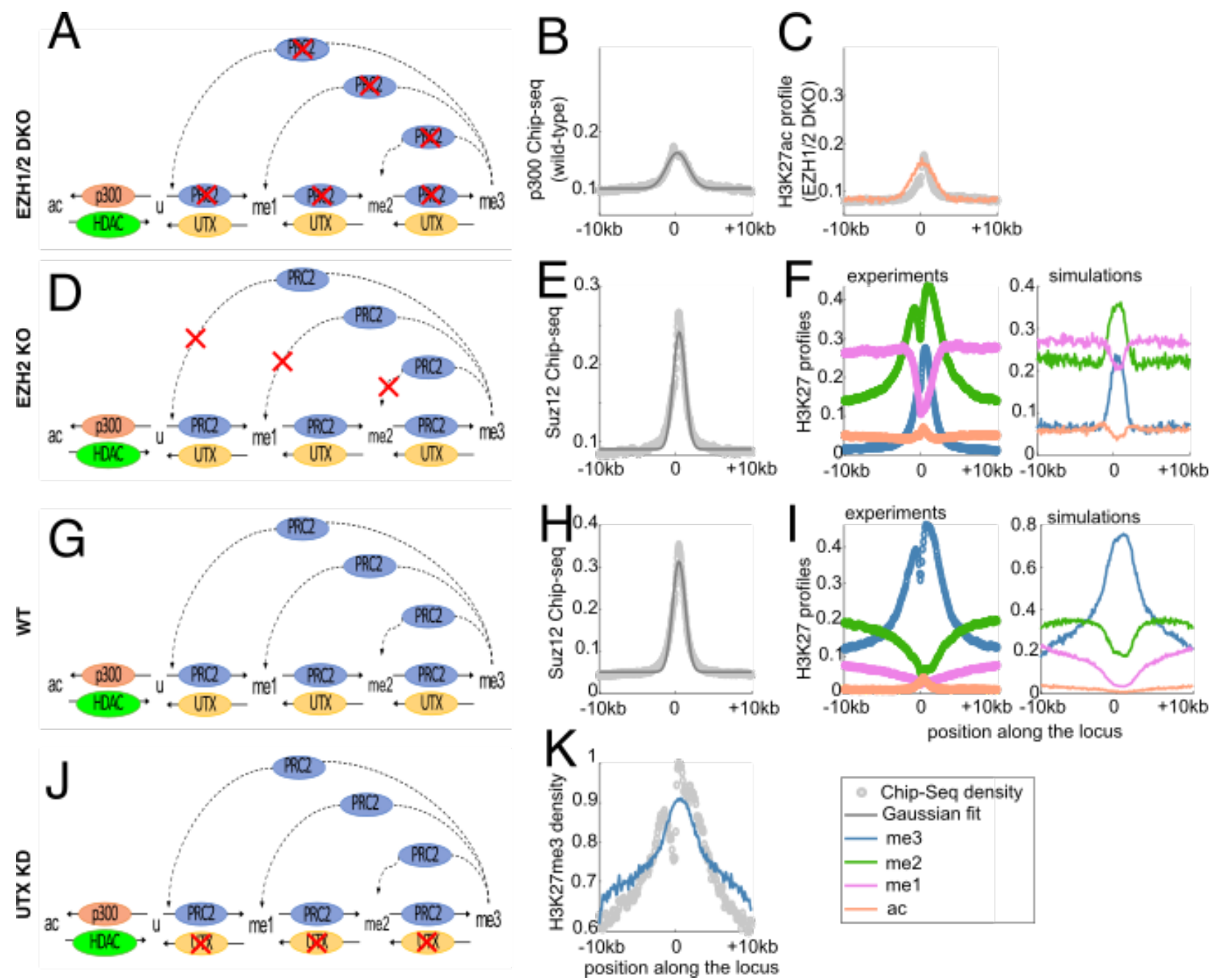

Figure 2: Fitting the model with various perturbation experiments around PcG-target genes. All profiles in the figure are around TSS (position 0) of PcG-target genes. (A,D,G,J) Schematic representation of the epigenetic models used to simulate EZH1/2 DKO, EZH2KO, WT and UTX KO cases. (B) Average p300 occupancy in WT. (C) Fit of the average H3K27ac profile in EZH1/2 DKO cells. (E,H) Average SUZ12 occupancy for the EZH2KO $(E)$ and WT $(H)$ cases. $(F, I)$ Experimental (left) and simulated (right) profiles of H3K27 marks for the EZH2KO (F) and WT (I) cases. (K) Fit of the average H3K27me3 profile for the UTX $\mathrm{KO}$ condition. In $(\mathrm{B}, \mathrm{C}, \mathrm{E}, \mathrm{F}, \mathrm{H}, \mathrm{I}, \mathrm{K})$, circles correspond to normalized Chip-Seq profiles (grey for HMEs, colored for H3K27 marks), grey full lines to gaussian fits of the HME profiles and colored full lines to the predicted profiles of the epigenetic states.

\section{Inference of methylation-related rates using EZH2 KO, WT and UTX KD profiles}

To infer the methylation-related parameters of the model, we designed an iterative scheme (Fig.2D-K) by sequentially using data from EZH2 KO, wild-type and UTX KD cells (see Materials and Methods section for details) for fixed ratios $r_{13} \equiv k_{m e 1} / k_{m e 3}$ and $r_{23} \equiv k_{m e 2} / k_{m e 3}$. (i) We started by initializing the nucleation rate $k_{m e 3}$ to an arbitrary value. (ii) Then, we took advantage of available data for EZH2 KO cells (29). Indeed, in this strain, while the average PRC2 occupancy is maintained (Fig.2E), PRC2 loses its allosteric long-range spreading capacity (Fig.2D). This 
leads to a drastic reorganization of the methylation landscape (Fig.2F) with $m e_{2}$ becoming the dominant methylation state in a $5 \mathrm{kbp}$-large portion surrounding the TSS (compared to the wildtype case on Fig.2I). By fitting this change of valency using a simplified version of the model with $\epsilon_{m e 1 / m e 2 / m e 3}=0$ (no long-range spreading), we could infer the demethylation rate $\gamma_{m e}$. (iii) Next, we reintroduced the spreading parameters (Fig.2G) and considered the wild-type profiles (Fig.2I) to fit the spreading-vs-nucleation ratio $R$ based on the methylation valency around promoters. (iv) After this step, there were no more free parameter in the model, however inferred $\gamma_{m e}$ and $R$ values may depend on the initial guess made for $k_{m e 3}$ at stage (i). We thus used an independent dataset to validate our full set of parameters. For this, we compared the quantitative H3K27me3 profile given by MINUTE Chip experiment for UTX KD cells (32) (Fig.2K) to the model prediction where active demethylation by UTX has been inhibited $\left(\gamma_{m e}=0\right)$ (Fig.2J). If not consistent, the same data allowed us to correct and optimize the $k_{m e 3}$ value, keeping all the other parameters fixed. By repeating steps (ii)-(iv) for this corrected value, we reevaluated $\gamma_{m e}, R$ and possibly $k_{m e 3}$, until convergence (Fig.S1).

This overall inference strategy was then applied to several values for $r_{13}$ and $r_{23}$ (Table S1). Over all the tested cases, only one pair of ratios $\left(r_{13}=3, r_{23}=3\right)$ lead to the convergence of the inference scheme and to an overall acceptable goodness of fit (Fig.S1,2). Qualitatively, such ratios' values are consistent with in vitro experiments on human EZH2 (50) showing a differential activity of PRC2 on $\mathrm{H} 3 \mathrm{~K} 27 \mathrm{u}$, me1 or me2 substrates with faster methylation rates towards me1 and me2 states than towards me3 $\left(r_{13} \geq r_{23} \geq 1\right)$. Quantitatively, our estimation suggests that, in vivo, addition of the third methyl group (me2 to me3 transition) is a rate-limiting step for chromatin to acquire a H3K27me3 - repressed - state, but at least 2 or 3 times less that initially observed in vitro (50). The other optimal parameters are $k_{m e 3}=0.81 h^{-1}, R=0.85$ and $\gamma_{m e}=1.5 h^{-1}$ (Table 1). Interestingly, such close-to-one value for the spreading-vs-nucleation parameter $R$ suggests that the allosteric boost of the EZH2 spreading efficiency mediated by H3K27me3 is of the order of 5- to 10-fold (see Materials and Methods), in very good agreement with in vitro experiments on human EZH2 (44,66).

\section{PRC2 spreading efficiency dictates the shapes, valencies and correlations of methylation profiles}

Our inference process illustrates how the epigenetic model and the underlying mechanistic hypotheses may consistently reproduce the profiles of all H3K27 marks around PcG-target genes. Remarkably, while parts of the inference are based on qualitative fits of the average methylation valencies around the promoter, the model predicts quantitatively the inversion of valency occurring far from the promoter in wild-type and in EZH2KO cells (Fig.2 F-G and J-K). We thus wondered what is the main mechanism driving such inversion. By varying the value of the spreading-vs-nucleation ratio $R$ while keeping HME profiles and other parameters as in the WTcase, simulations strongly suggest that valency around PcG genes is mainly driven by the longrange, allosteric spreading capacity of PRC2 (Fig.3A). When $R$ is very low (EZH2KO-like situation, $R \sim 0$ ), me2 dominates at the nucleation sites (Fig.3A, TSS $<2.5 \mathrm{kbp}$ ) while me1 is predominant in the rest of the region (Fig.3A for distances to TSS> $2.5 \mathrm{kbp}$ ). Interestingly, in this low- $R$ regime, while the proportions of methylation states are overall limited, the model predicts that the levels 
of H3K27 acetylation are still low (less than 10\%) everywhere and therefore we may expect that most of the PcG-target genes should remain inactive. This is in line with experimental observations showing that the massive transcriptional deregulation of PcG-target genes observed in $\mathrm{EZH} 1 / 2$ DKO cells is already rescued in EZH2 KO cells (31). As $R$ increases, we progressively observed the emergence of H3K27me3 as the dominant state. The increase in me3 follows a switch-like, sigmoidal, function as a function of $R$ (blue lines in Fig.3A), signature of a phasetransition driven by the allosteric spreading. Strikingly, the inferred parameter for WT $(R=0.85$, red dotted lines in Fig.3A) lies in the transition zone between the low and the high me3 regimes.
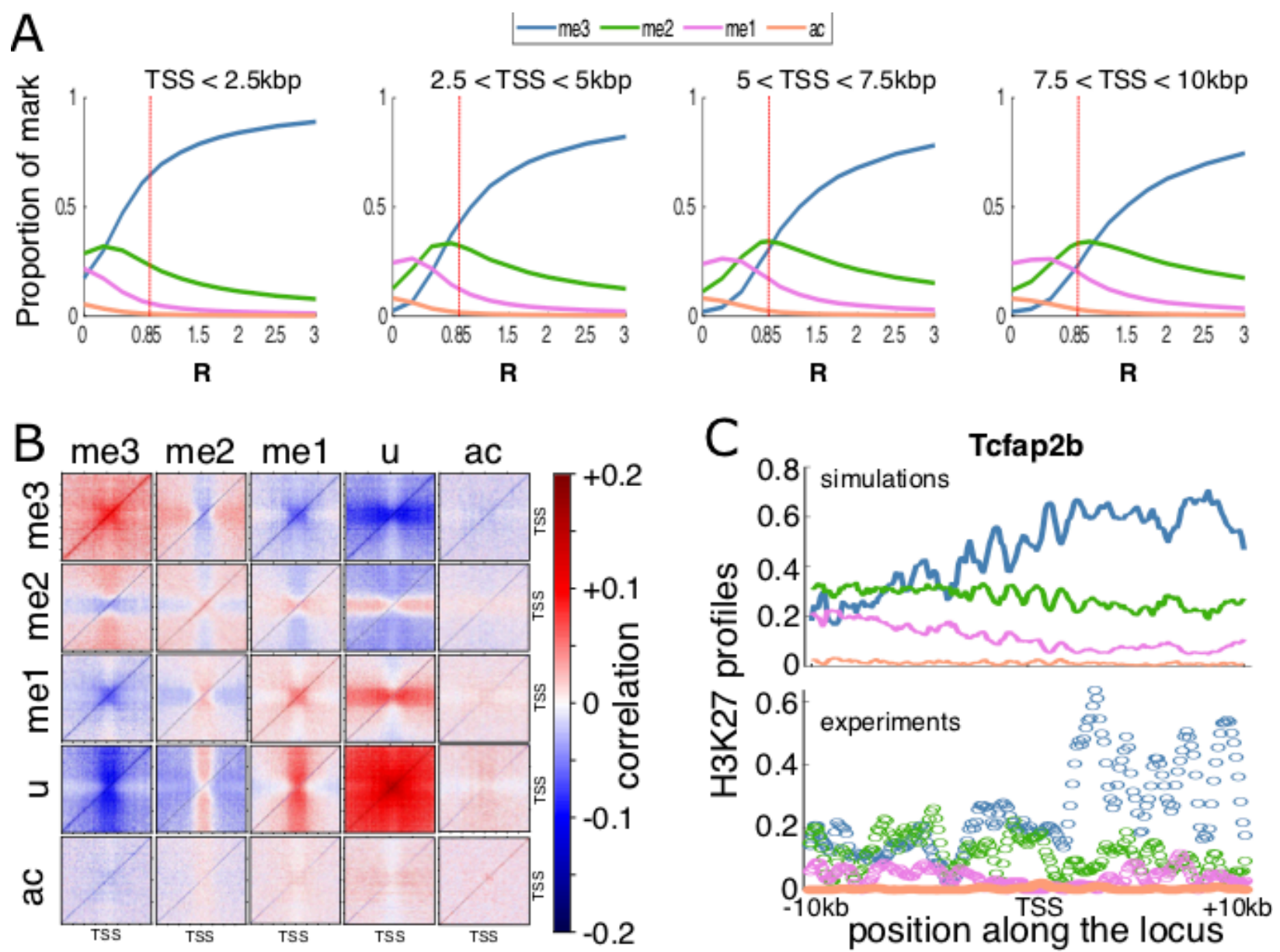

Figure 3: Effect of spreading efficiency on PcG-target genes. (A) Average predicted proportion of a given mark as a function of the spreading-to-nucleation ratio $R$, all other parameters fixed to WT. Panels from left to right correspond to regions close or far from TSS. (B) Correlation between H3K27 states at different positions around TSS for the WT parameters. (C) Predicted (top) and experimental (down) WT profiles around the gene Tcfap $2 b$.

As the spreading mechanism is constrained by the presence of $\mathrm{H} 3 \mathrm{~K} 27$ me 3 marks at the binding sites of PRC2, we expected to observe long-range correlations between the H3K27me3 level around the TSS where HMEs bind and the methylation state at more distal regions. More generally, to estimate the co-occurence of H3K27 states at different positions, we computed from the simulated stochastic trajectories (Fig.S3) the correlations (see Materials and Methods) between the instantaneous local epigenomic state of any pairs of loci in the WT situation. Fig.3B 
illustrates the complex pattern of correlations existing between states and loci. As expected, positive correlations are observed between H3K27me3-tagged loci. Acetylated loci are weakly negatively (respectively positively) correlated with highly (resp. lowly) methylated states me2/3 (resp. me0/1). Due to the 'synchronized' dilution happening at replication, H3K27u-tagged loci are highly correlated. Other correlations between states translate the local competition between them and the long-range spreading capacity of H3K27me3 states at TSS. For example, me2 at (resp. out of) TSS is negatively (resp. positively) correlated with me3 everywhere. Indeed, me2 at TSS does not allow spreading methylation while me2 out of the nucleation region is the pathway towards me3 via spreading by me3 from TSS.

So far, we have parameterized and analyzed our model using the average experimental densities of HMEs around PcG genes as inputs and the corresponding average experimental profiles of $\mathrm{H} 3 \mathrm{~K} 27 \mathrm{ac} / \mathrm{me} 1 / \mathrm{me} / \mathrm{me} 3$ modifications as targets. Therefore, we wanted to test whether the same parameters are also viable for individual genes. Overall, plugging HME densities of individual genes into the simulations, we found that the individual profiles for each modification as well as the methylation valencies are well captured by the model (see examples in Fig.3C and Fig.S4). This is remarkable that we can still reproduce the specificity of each gene knowing that our parameterization has been based on an average signal that smoothed out these specificities.

\section{Parameter-free predictions of H3K27 modifications at active and bivalent genes}

Active genes
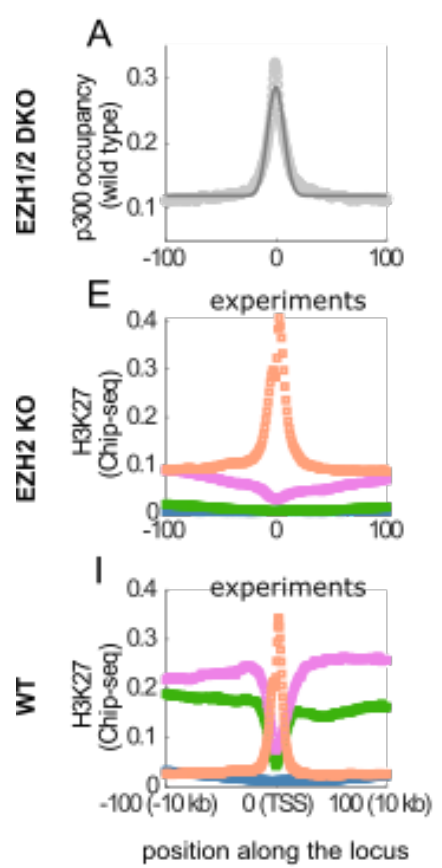

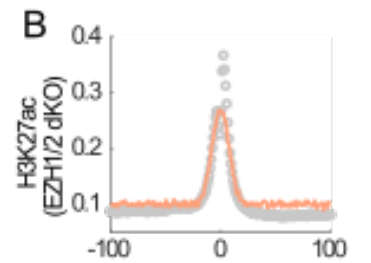

$\mathrm{F}$
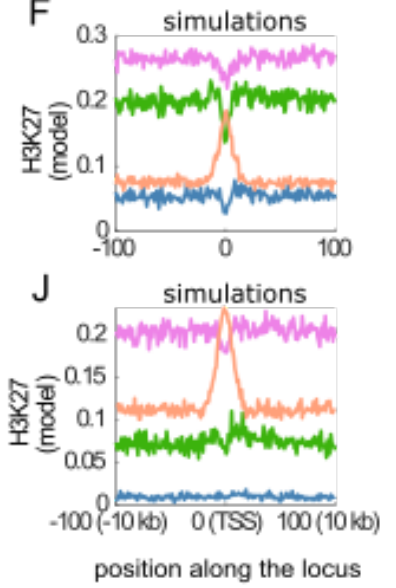

C

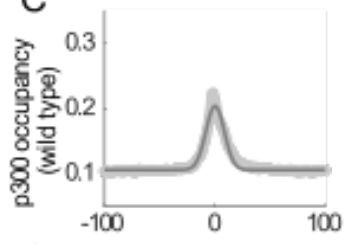

G

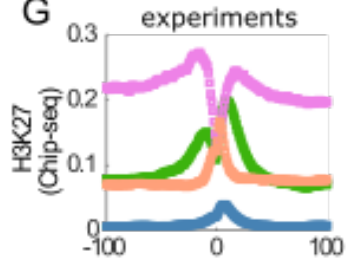

$\mathrm{K} 0.3$ experiments

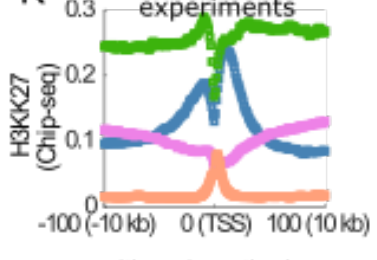

position along the locus

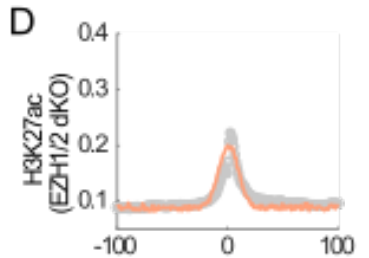

$\mathrm{H}$

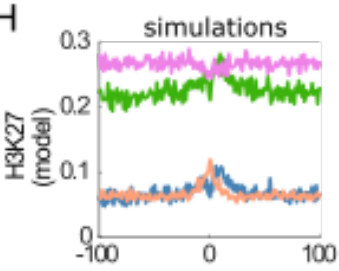

$\mathrm{L}$
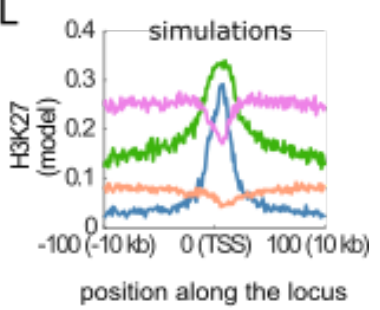

Chip-seq density - Gaussian fit - me3 - me2 - me1 - ac

Figure 4: Predictions of H3K27 modifications for active and bivalent genes. (A,C) Average p300 occupancy in WT around active (A) and bivalent (C) genes. (B,D) Predictions of the average H3K27ac profile in EZH1/2 DKO cells for active (B) and bivalent (D) genes. $(E-L)$ Experimental $(E, G, I, K)$ and 
simulated $(\mathrm{F}, \mathrm{H}, \mathrm{J}, \mathrm{L})$ profiles of H3K27 marks for the EZH2KO $(\mathrm{E}-\mathrm{H})$ and $\mathrm{WT}(\mathrm{I}-\mathrm{L})$ cases around active $(E, F, I, J)$ and bivalent $(G, H, K, L)$ genes. Circles correspond to normalized Chip-Seq profiles (grey for HMEs, colored for H3K27 marks), grey full lines to gaussian fits of the HME profiles and colored full lines to the predicted profiles of the epigenetic states.

In our epigenetic model, the various methylation or acetylation patterns observed at different loci emerge from the differential binding of HMEs at these regions. We therefore asked if the shapes and valencies of H3K27 modifications observed around active and bivalent genes $(25,29)$ may be predicted by the model using the same parameters as previously inferred at PcG genes but with the active and bivalent average HME profiles.

In EZH1/2 DKO cells, the model is able to predict quantitatively the acetylation profiles of bivalent and active genes using their respective corrected p300 profiles (Fig. 4A-D). In EZH2 KO cells, the model well predicts the methylation valencies for both active and bivalent genes but fails in capturing the acetylation level (Fig.4E-F), suggesting that the p300/UTX profiles that we took from WT as they were not available in the mutant strain, may be strongly perturbed in EZH2KO around non-PcG genes with a higher occupancy as observed in EZH1/2 DKO cells.

In the WT case, active genes exhibit an inverse H3K27 methylation landscape compared to PcG domains with a valency me1 > me2 > me3 (Fig.4H). Interestingly, the average HME densities are also inverse with those around PCG genes: poor PRC2 binding and rich UTX/p300 occupancy (Fig.S5). Using these profiles as inputs, the model is able to well predict the average histone marks profiles including the observation that acetylation is higher than the methylation levels at the promoter (Fig.4I), the behavior around individual genes being also well captured (Fig.S6). At bivalent genes, H3K27me3 has the almost same peak density (average) as H3K27me2 around the promoter $( \pm 2.5 \mathrm{kbp}$ ), both being higher than H3K27me1 and ac (Fig.4J). Our epigenetic model performs reasonably well in this region (Fig.4K) even if prediction for H3K27me3 is slightly lower than observed (see also Fig.S7). However, the model completely fails for more distal regions where ChipSeq experiments show for example a flat profile for H3K27me2 that we don't capture. This suggests that other mechanisms not included in the model might play an important role at bivalent genes. For example, activating marks like $\mathrm{H} 3 \mathrm{~K} 4 \mathrm{me}$ or $\mathrm{H} 3 \mathrm{~K} 36 \mathrm{me}$, also present at bivalent genes $(32,33)$, may interfere with the H3K27 dynamics $(16,59)$.

\section{Competition between activating and repressing factors shapes the local epigenomic landscape}

More generally, we asked how the differential recruitment of HMEs around TSS may impact the local epigenetic landscape and subsequently gene regulation. To investigate this, we systematically computed the average profiles of H3K27 modifications as a function of the recruitment strengths of $\mathrm{p300/UTX}$ and PRC2 (see Materials and Methods). For each recruitment condition, we estimated the methylation valencies around TSS (Fig.S8) and found that the phase diagram of the system can be divided into 3 qualitative behaviors (Fig.5): PcG-target-like landscapes with me3>>me2>me1 for 'high' PRC2 and 'low' p300/UTX recruitments, active-like landscapes with me1>me2>>me3 for 'low' PRC2 and 'high' p300/UTX recruitments, and other 
types of landscape including bivalent-like cases (me2 me3>me1) for intermediate situations. The frontiers between these regions are almost straight lines with a stiffer dependency to the PRC2 occupancy, signature of the asymmetric tug-of-war between the acetylation by p300 and demethylation by UTX from one side and the methylation by PRC2 on the other side. The levels of p300/UTX at an average PcG-target gene in mESC (blue dot in Fig.5A) would need to be 3 times higher than the levels typically observed around active genes in mESC (orange dot in Fig.5A) to switch the gene into the active area for the same PRC2 level; while the level of PRC2 at an average active gene in $\mathrm{mESC}$ should be increased by $80 \%$ of the typical level found at PcGtarget genes in $\mathrm{mESC}$ to move the gene into the PcG area. This suggests that activation of former PcG-target genes, during differentiation for example, at more reasonable levels of p300/UTX would require a concomitant decrease in PRC2 occupancy in parallel to the increase in p300/UTX recruitments. As repressors (PRC2) and activators (p300/UTX) binding motifs or recruitment signals are usually colocalized around TSS (67), a competition for their bindings to chromatin may naturally cause the inhibition of repressor occupancy while activator binding increases (or vice versa) (17).
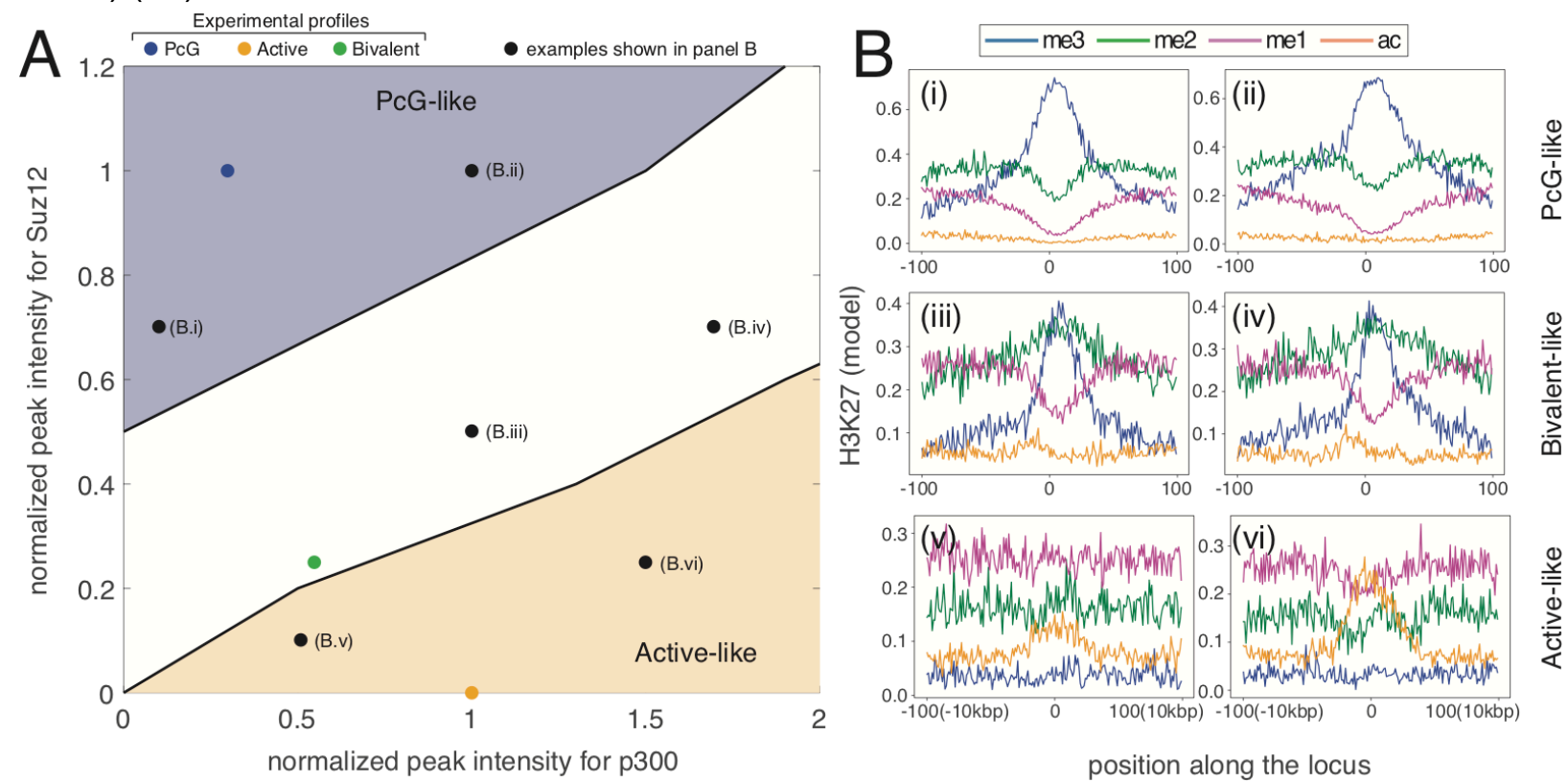

Figure 5: Differential recruitment of HMEs at nucleation sites. (A) Phase diagram of the model behavior obtained by varying the strengths of recruitment of p300 (x-axis) or Suz12/PRC2 (y-axis) around TSS for WT parameters. P300 (Suz12) peak intensities are normalized by the corresponding value at active (PcGtarget) genes. The blue area represents situations where the methylation valency around TSS is PcGtarget-like (me3>>me2>me1), the orange area to active-like conditions (me1>me2>>me3), the white zone to other cases including bivalent genes (me2 me3>me1). Colored dots give the positions of WT experimental profiles studied in Fig.2 and 4. Black dots are other special examples shown in panel (B). (B) Predicted epigenetic state profiles at different positions in the phase diagram (black dots in (A)). 


\section{The model captures the maintenance and spreading dynamics of H3K27me3}

Previously, we showed that the model well captures the average, 'static' epigenetic landscape around genes for a population of asynchronized cells. We finally sought to use it to investigate the dynamics of regulation of H3K27 modifications.

We first interrogated how the epigenetic landscape is re-established after the strong, periodic perturbation of the system occurring every cell cycle at replication where half of the epigenetic information is lost. With the WT parameters inferred above, focussing on PcG-target genes, we tracked the dynamics of each H3K27 marks after replication for a population of synchronized cells in a periodic steady-state (Fig.6A). The me1 level rapidly increases up to $\sim 4$-fold (reached at $~$ $T / 7$ ) and then slowly decays by 2 -fold towards its pre-replication value. The me2 profile reaches almost its pre-replication value after $\sim T / 3$. The me3 level slowly grows along the whole cell cycle. This dynamics translates the gradual and slow re-establishment of H3K27me3 marks from the unmarked histones newly integrated at replication that are rapidly methylated to me1, which represents a transient state towards higher methylation states. Even if me2 reaches plateau suggesting that the 'me1 to me2' and 'me2 to me3' fluxes equilibrate, the system as a whole never reaches a steady-state during cell cycle due to the 'me2 to me3' rate-limiting step that occurs more slowly than the other transitions $\left(r_{13}=3, r_{23}=3\right.$, see above). Remarkably, our predictions are in qualitative agreement with the cell cycle dynamics of whole-cell contents of H3K27me1/me2/me3 measured in human HeLa cells using SILAC (Fig.6B) (60). The model even captures the small decrease in H3K27me3 level just after replication, that in our model can be interpreted by a significant demethylase activity not yet compensated by the methylation flux from the me2 state.

To better characterize the dynamics of newly integrated histones in the maintenance of a stable epigenetic landscape, we turned to a simpler system of unsynchronized cells where, we tracked the time-evolution of the epigenetic state of unmarked histones incorporated in the region after a given time $t_{0}$ due to histone turnover or replication (scheme at the top of Fig.6C,E). For this pool of 'new' histones, we observed the same type of dynamics than along the cell cycle: me1 has a transient dynamics, me2 reaches a plateau and me3 grows very slowly (Fig.6E), confirming that the establishment of me3 marks on new histones extends over a long period $(\sim 2 T)$. Consistently, the proportion of me 3 in the pool of 'old' histones that were integrated before $t_{0}$ is still slowly increasing after $t_{0}$, while me2 levels remain almost constant and the me1 content slightly decreases (Fig.6C). Again, both predictions on old and new pools are qualitatively consistent with recent SILAC experiments performed on mESC (Fig.6D,F) (59), the recovery rate of H3K27me3 being even slower in the experiments than predicted.

Finally, we analyzed the spreading dynamics of H3K27me3 around PcG-genes. We prepared the system as a population of unsynchronized cells evolving with a $\mathrm{EZH} 2$ inhibitor $(R=0$, no spreading) (scheme at the top of Fig.6G,H). Then, at a given time $t_{0}$, the inhibitor is washed out ( $R=0.85$, WT parameters) and we tracked the establishment of the epigenetic landscape after $t_{0}$ (full lines in Fig.6G, top). Due to the inhibition of $E Z H 2$, at $t=t_{0}$, the global level of me1 is higher 
than in normal WT condition (dashed lines in Fig.6G, top), while me2 and me3 are less present (see also Fig.2F,I). For $\mathrm{t}>t_{0}$, we observed that the spreading of $\mathrm{H} 3 \mathrm{~K} 27 \mathrm{me} 3$ and the recovery towards the WT state is slow and takes few cell generations $(\sim 2 T)$. These predictions are in perfect agreement with similar experiments on mESC studied with SILAC (Fig.6G, bottom) (59) or Chip-Seq (31) but also with EED-KO rescue experiments in $\mathrm{mESC}$ showing a recovery of the WT levels about 36 hours after the rescue (6). After the release of the inhibition, we also followed the dynamics of newly incorporated histones (full lines in Fig.6G) and compared it to the dynamics of new histones but in WT conditions (Fig.6E and dashed lines in Fig.6G). Overall, we observed a shift of $\sim T / 5$ in the establishment of the epigenetic identity of the new histones compared to WT, consistently with SILAC experiments (59). This delay is imputable to the weak density of $\mathrm{H} 3 \mathrm{~K} 27$ me3 marks at the nucleation sites at $t_{0}$ that limits initially the long-range spreading by $\mathrm{PRC} 2$. This highlights the role of pre-existing $\mathrm{H} 3 \mathrm{~K} 27 \mathrm{me} 3$ in controlling the dynamics of de novo methylation states thanks to the reader-writer capacity of PRC2.

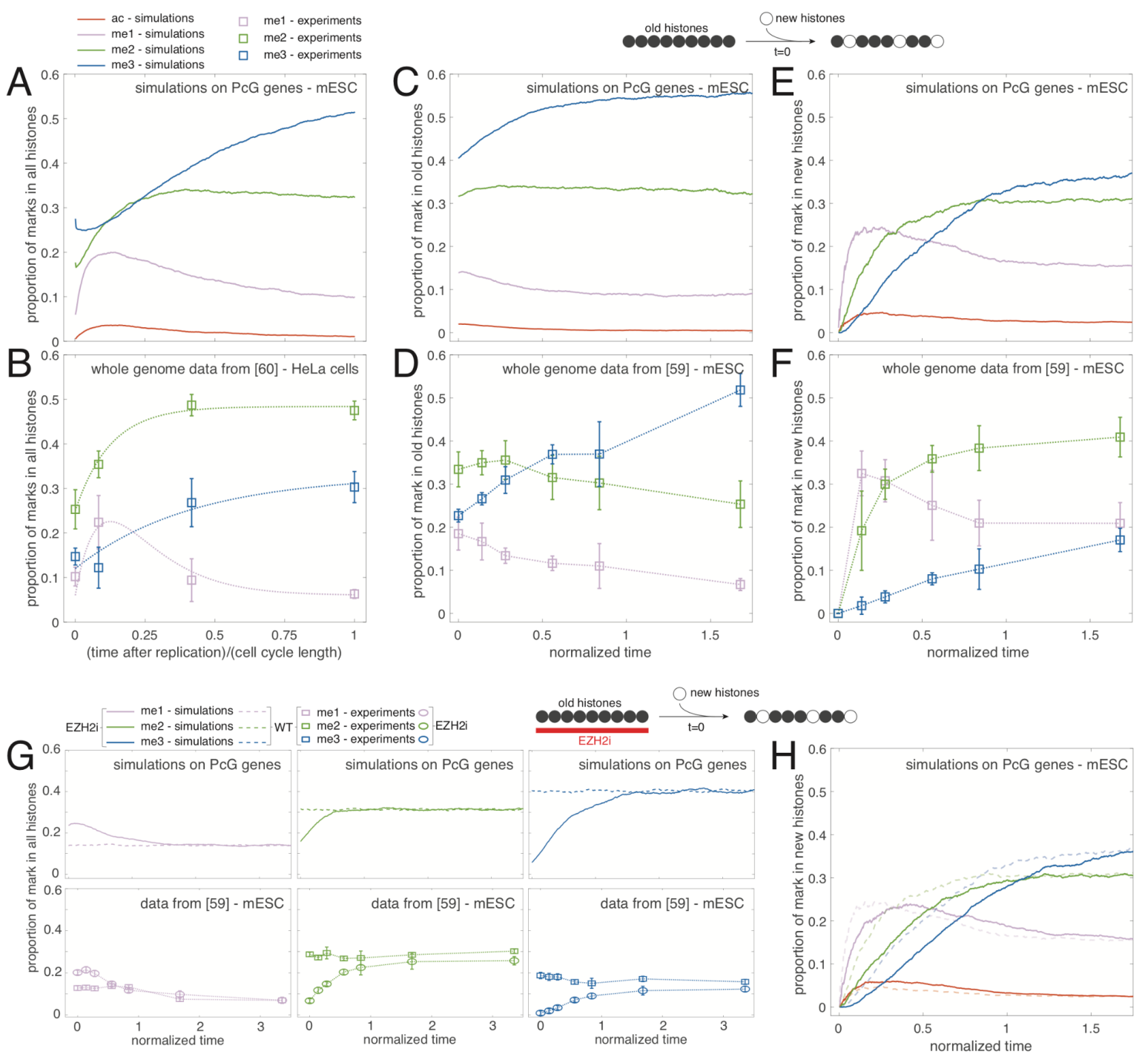

Figure 6: Dynamics of H3K27 modifications. (A,B) Intra-cell cycle dynamics for a population of synchronized cells as a function of the time after the last replication, predicted by the model for the WT 
parameters averaged around mESC PcG-target genes (A) or measured by SILAC experiments (B) on HeLa cells (data extracted from (60), see Materials and Methods). For each cell type, time is normalized by the corresponding cell cycle length ( $T=13.5 \mathrm{~h}$ for mESC simulations, $T=24 \mathrm{~h}$ for HeLa cells). Dotted lines in (B) are a guide for the eye. (C-F) In WT conditions, we tracked from a given time $(t=0)$ the dynamics of marks in the pools of (E, F) newly incorporated (after turnover or replication) and (C,D) remaining (old) histones, for a population of unsynchronized cells. Predictions for the WT parameters averaged around mESC PcG-target genes are given in (C, E); SILAC experiments on mESC in (D,F) (extracted from (59), see Materials and Methods). Time is normalized by the effective histone decay time $\left(t_{e}=12.7 \mathrm{~h}\right.$ for simulations, $t_{e}=28.6 \mathrm{~h}$ for experiments) (see Materials and Methods). (G, H) A population of unsynchronized cells is first evolved in presence (EZH2i) or absence (WT) of an EZH2 inhibitor. Then, at $t=0$, if present, the inhibitor is washed out and we tracked the dynamics of marks in all histones $(G)$ or in newly incorporated histones after $\mathrm{t}=0 \mathrm{(H})$. Predictions for the WT parameters averaged around $\mathrm{mESC}$ PcGtarget genes are given in (G, top; H); SILAC experiments on mESC in (G, bottom) (extracted from (59), see Materials and Methods). Time is normalized as in (C-F).

\section{Discussion and conclusion}

In this work, we developed a model which accounts for the recruitment of HMEs at a domain of interest and then determines the histone modification levels as a consequence of a complex competition between the spreading and erasing capacities of these HMEs. In the light of rich quantitative data available from recent experiments, we picked up the case of H3K27 modifications in mESCs which allows, by modeling one residue, to investigate Polycombrepressed, active and bivalent genes at the same time. By integrating key mechanistic details like the reader-writer capacity of PRC2 and experimental data of HME occupancy (Fig.1), this framework allowed us to analyze different conditions under one umbrella.

In particular, we inferred model parameters using data from WT and mutant conditions (EZH1/2 DKO, EZH2 KO, UTX KD) around PcG-target genes (Fig.2). We found that, to reach the repressive H3K27me3 state, the me2 to me3 transition was the limiting time step (50). Our strategy also highlights the importance of looking at the full density profiles and methylation valencies around TSS (or nucleation sites) to efficiently estimate the spreading activity of PRC2. In particular, we estimated that the 'writing' efficiency of PRC2 is boosted by 5- to 10-fold when bound to H3K27me3 histones (44).

Our analysis suggested that such long-range, enhanced mechanism drives a transition between a low and high me3 regime and is essential for maintaining a proper H3K27me landscape (Fig.3A). The sigmoidal shape of this transition suggests that the epigenetic landscape could be sensitive to variations in the spreading efficiency (9). While this may be advantageous for WT embryonic cells that are plastic and may need to differentiate rapidly following developmental cues, perturbations of this key allosteric capability may have deleterious impacts on gene expression. For example, gene deregulation in pediatric gliomas is associated with a loss of the $\mathrm{EZH} 2$ allosteric stimulation, mediated by the interactions between PRC2 and the oncohistone H3K27M or the oncoprotein EZHIP (68).

While the model was parameterized using average data around PcG-target genes, it was able to predict semi-quantitatively the H3K27 densities around individual PcG-target genes, but also around active and bivalent genes, by only plugging in the corresponding HMEs profiles (Fig.4). A systematic analysis of the role of HME recruitment (Fig.5A) allowed us to characterize the 
competition for epigenetic control between active and repressive factors. We showed that genes can be categorized as repressed, active or bivalent depending on the levels of recruitment of activators and repressors. In particular, we observed that PRC2 binding at promoters, even at mild degrees, is essential to avoid spurious transcription by increasing the level of P300/UTX recruitment needed for transcriptional activation (14,33). Activation of PcG-target genes should be necessarily accompanied by a significant decrease in PRC2 binding via, for example, the competition with activators for the binding at promoters $(17,69)$. Moreover, looking at the consistency between the predicted category of expression (silenced, active, bivalent) and the observed one may be used to identify genes that are under the direct control of the H3K27 marks and associated HMEs.

Beyond the 'static', average description of H3K27 profiles around TSS, the model can be used to predict the dynamics of maintenance or establishment of the epigenetic landscape (Fig.6). In perfect agreement with experiments $(59,60)$, we observed that the (re)formation of H3K27me3 domains de novo or after replication is a slow process in cycling mESCs. This indicates that regulation of the cell cycle length during embryogenesis for example may also impact the stability and plasticity of the epigenetic landscape $(17,70)$. We showed that the reader-writer capacity of PRC2 strongly influences such dynamics at PcG-target genes. Simply put, PRC2 which is recruited at promoters, first tri-methylates histones $\mathrm{H} 3 \mathrm{~K} 27$ at these nucleation sites, and then, thanks to allosteric activation, can tag more distal sites and spread methylation. The initial presence of $\mathrm{H} 3 \mathrm{~K} 27 \mathrm{me} 3$ thus accelerates this process. This suggests that defects in partitioning of maternal H3K27me3 histones between the leading and lagging daughter strands $(61,62)$ may generate asymmetries that may propagate to further generations as the H3K27me3 recovery dynamics is slow (71).

Previous generic models of epigenetic regulation $(8-11,17)$ suggested mathematically that the maintenance of a robust and plastic epigenetic state may be associated with bistability that emerges from the self-propagation capacity of some epigenetic marks (8,9). Here, we proposed, in line with recent experiments for the PcG system $(6,31,72)$, that robustness is associated with the stable recruitment of HMEs at specific nucleation sites coupled to the long-range, allosterically-boosted spreading capacity of PRC2. As it is, our model cannot lead to mathematical bistability: PcG-target-like repression and bivalency are not bistable states (16) in our framework but rather correspond to a bimodal and a highly fluctuating state, respectively (Fig.S9).

Compared to the few other explicit models of PcG regulation in mammals $(14,18,25)$, our framework also integrates a spreading process in competition with antagonistic erasing and activating processes, but the mechanistic nature of spreading differs. Chory et al (25) did not account for the allosteric enhancement of PRC2 and hypothesized that spreading from the nucleation site occurs via histone exchange between nearest-neighbor (NN) nucleosomes. Berry et al (14) considered the allosteric boost and that any H3K27me3 histone can spread methylation to its NN sites, allowing bistability to emerge. In terms of spreading, our formalism is closer to Erdel et al (15) that modeled $\mathrm{H} 3 \mathrm{~K} 9 \mathrm{me} 3$ regulation in $S$. pombe from the methylation long-range activity of enzymes bound at a nucleation site.

In addition to the richness of available datasets, by modeling H3K27 regulation in mESCs, we were hoping that the high plasticity of these cells would allow us to explain their epigenetic 
landscape by simply focusing on main, primary mechanisms while neglecting a priori secondary effects. Our good description of the H3K27 profiles in different conditions around PcG-target genes and, to a lesser extent, around active and bivalent genes validated our approach. However, our predictions also contained discrepancies suggesting missing ingredients that may improve the description of H3K27 regulation in mESCs but also in more differentiated cell types.

A strong hypothesis that we made is that $\mathrm{H} 3 \mathrm{~K} 27$ profiles are only readouts of the HME occupancies via a complex network of interactions, but they do not feedback on the binding of HMEs or on the model parameters like UTX activity (47) or the histone turnover rate that may be impacted by transcription $(14,25,73,74)$. For example, the PRC2 'reader' subunit EED is known to interact with $\mathrm{H} 3 \mathrm{~K} 27 \mathrm{me} 3$ histones (44). We assumed that such interaction was only relevant at nucleation sites to enhance EZH2 activity, but it may also lead to the recruitment of PRC2 at more distal sites and allow the self-propagation of the H3K27me3 mark (66). We expect however such an effect to be weak in mESCs as the profile of H3K27me3 around TSS is much larger than the PRC2 binding density (Fig.2H,I) and a significant self-propagation would have led to profiles with more similar shapes. To account for such feedback, one would need to model explicitly the binding and unbinding of HMEs. This would allow also to describe in more detail the role of PRC1 variants in nucleation and maintenance (35-37) or the competition for the binding of antagonistic HMEs around the same site (17).

Another simplification made in our work was to only model H3K27 modifications. While this might be sufficient to describe the regulation of PcG-target genes, a more acute description of active and bivalent genes may require accounting for other 'active' modifications like the methylations of H3K4 and H3K36 by Trithorax-group proteins $(16,59,75)$ that may interfere with H3K27me states (75).

In our formalism, the long-range spreading of methylation results from 3D contacts between nucleation and distal loci. In this work, we assumed that such contacts were dependent only on the genomic distance and were not affected by the local epigenetic landscape. However, PRC1 that binds to H3K27me3 may form condensate $(45,76-78)$, the so-called Polycomb bodies, and may subsequently impact the local 3D organization of the locus $(79,80)$, an increase compaction may in turn facilitate spreading (81-83). Accounting for this positive feedback loop between longrange spreading and $3 \mathrm{D}$ chromosome organization may allow a better characterization of the role of genome folding in epigenetic regulation $(84,85)$.

To conclude, the ideas and formalisms developed here are general in nature and are adaptable to other cell types or epigenetic systems. In particular, it would be interesting to investigate the generality of the inferred parameters for more differentiated cells where H3K27me3 domains are usually more extended around PcG-target genes than in mESCs. It would allow to understand if these changes are solely due to differential HME binding, to modifications of rates like histone turnover $(73,86)$ or to some unconsidered mechanisms as discussed above. More generally, our approach represents a first step towards a quantitative description of PcG regulation in various cellular contexts where 'secondary' effects may be integrated step-by-step to better estimate their importance in normal or disease contexts. 


\section{Materials and methods}

\section{Chip-seq data analysis}

We collected the raw Chip-seq data of various histone modifying enzymes (SUZ12, p300, UTX) and of H3K27-me3/me2/me1/ac marks from various sources as listed in Table 2. Corresponding fastq files were imported in the Galaxy environment (87) and mapped using bowtie2 (88) to the mouse genome (mm9). For ChipSeq data with spike-in control, reads were mapped to the combined mouse + drosophila genomes $(\mathrm{mm} 9+\mathrm{dm} 6)$. After removing the duplicates and sorting the bam files using samtools (89), reads were normalized by the total number of mm9 mapped reads for normal Chip-seq and by the one of dm6 for Chip-seq with spike-in (90). While utilizing all of these tools, we made sure that the same settings as in the original papers were used. To analyze the Chip-seq data, we first used bamcoverage from the deepTools 2.0 suite (91) to generate genomic profiles at a binning of $50 \mathrm{bp}$. Then, to make quantitative comparisons between different histone modification levels, each profile was further normalized (25) using $\mathrm{R}$ by dividing each bin value by the maximal value of the bin count over the genome, this maximum being estimated after removing outliers (bins outside the quantile range $(0.1 \%, 99.9 \%)$ ). Although the above normalization method allows for comparison across different antibodies (25), we want to make it clear that these are not absolute probabilities a priori. Normalized average profiles around TSS for PcG-target, bivalent and active genes were computed from the matrix files given by computeMatrix (deepTools 2.0) (91) for each list of genes that we took from (25).

The above method was used for normal ChipSeq data except for the UTX/JMJD3 inhibited experiment obtained with MINUTE Chip (32). In this case, the normalized bigwig file was directly sourced and fed to computeMatrix. Different from normal Chip-seq or Chip-seq spike-in experiment, the reads are normalized with total mapped reads of input to evaluate the input normalized read count (INRC). It was shown in (32) that the INRC can be regarded as a signal proportional to the true density of H3K27me3 histones over the genome. Here, we further assume that in absence of demethylase and demethylase-driven activation signals, genes targeted by Polycomb will attain maximum possible H3K27me3 levels. Then, the INRC at PcG-target genes is further scaled with the average peak density to approximate the actual density of H3K27me3 at PcG-target genes in UTX/JMJD3 inhibited mESCs. 
Table 2: List of Chip-Seq data used in this study

\begin{tabular}{|c|c|c|c|}
\hline Antibody & $\begin{array}{l}\text { Cell line and } \\
\text { perturbation }\end{array}$ & Experiment & Source \\
\hline $\mathrm{H} 3 \mathrm{~K} 27 \mathrm{me} 3 / \mathrm{me} 2 / \mathrm{m} 1 / \mathrm{ac}$ & Wild type mESCs-2i & Chip-seq Spike-in & \multirow[t]{5}{*}{ GSE116603 } \\
\hline SUZ12 & Wild type mESCs-2i & Chip-seq & \\
\hline $\mathrm{H} 3 \mathrm{~K} 27 \mathrm{me} 3 / \mathrm{me} 2 / \mathrm{m} 1$ & EZH2KO mESCs-2i & Chip-seq Spike-in & \\
\hline SUZ12 & EZH2KO mESCs-2i & Chip-seq & \\
\hline H3K27ac & $\begin{array}{l}\text { EZH1/2 DKO mESCs- } \\
2 \mathrm{i}\end{array}$ & Chip-seq Spike-in & \\
\hline p300 & $\begin{array}{l}\text { Wild type mESCs- } \\
\text { Serum }\end{array}$ & Chip-seq & GSM2417169 \\
\hline UTX & $\begin{array}{l}\text { Wild type mESCs- } \\
\text { Serum }\end{array}$ & Chip-seq & GSM2575693 \\
\hline H3K27me3 & $\begin{array}{l}\text { UTX/JMJD3 inhibited } \\
\text { mESCs-2i }\end{array}$ & $\begin{array}{l}\text { MINUTE } \quad \text { Chip } \\
\text { (calibration } \\
\text { experiment) }\end{array}$ & GSM3595377 \\
\hline
\end{tabular}

\section{Description of the stochastic epigenetic model}

\section{Mathematical formulation of the kinetic transition rates}

The dynamics of the epigenetic state is driven by kinetic rates accounting for the main features of the model described in the main text:

- Addition of one methyl group to histone $i$ to reach state $m e_{x}(x \in\{1,2,3\})$ is governed by $\left(m e_{0} \equiv u\right)$

$$
m e_{x-1}(i) \rightarrow m e_{x}(i)=k_{m e_{x}} \psi_{\text {suz } 12}(i)+\epsilon_{m e_{x}} \sum_{j} \frac{\psi_{\text {suz12 }}(j)}{(j-i)^{\lambda}} \delta_{j, m e 3}
$$

$k_{m e_{x}} \psi_{\text {suz12 }}(i)$ represents the local nucleation of the $m e_{x}(x \in\{1,2,3\})$ state with $k_{m e_{x}}$ the corresponding rate and $\psi_{\text {suz12 }}(i)$ the density of bound PRC2 at locus $i$. Practically, $\psi_{\text {suz12 }}(i)$ is given by the normalized Chip-Seq profile of Suz12 (see below). The right part in Eq.1 describes the long-range, allosteric spreading capacity of PRC2 with $\epsilon_{m e_{x}}$ the corresponding enzymatic activity and $\delta_{j, m e 3}=1$ if histone $j$ is trimethylated (=0 otherwise). This term depends on the probability of contact between two loci. To simplify, we assume a power-law contact model $1 /|j-i|^{\lambda}$, capturing the average polymeric behavior of chromatin (92), with $|j-i|$ the genomic distance between two histones $i$ and $j$, and $\lambda=1$ in accordance with Hi-C data in mESC (93).

- Removal of one methyl group to a $m e_{x}$ histone $(x \in\{1,2,3\})$ at position $i$ is driven by: 


$$
m e_{x}(i) \rightarrow m e_{x-1}(i)=\gamma_{m e} \psi_{U T X}(i)
$$

with $\gamma_{m e}$ the corresponding demethylation rates and $\psi_{U T X}(i)$ the density of bound UTX at locus $i$ that we extracted from the normalized ChipSeq data.

- Addition of acetylation at histone $i$ follows the propensity:

$$
u(i) \rightarrow a c(i)=k_{a c} \psi_{P 300}(i)
$$

with $k_{a c}$ the acetyltransferase activity of p300 and $\psi_{P 300}(i)$ the density of bound p300 at locus $i$ that we extracted from the normalized ChipSeq data.

- Removal of acetylation at histone $i$ is given by

$$
a c(i) \rightarrow u(i)=\gamma_{a c}
$$

with $\gamma_{a c}=0.6 h^{-1}$ the uniform deacetylation rate.

- Histone turnover leads to the loss of the current histone state replaced by a ' $u$ ' state:

$$
X(i) \rightarrow u(i)=\gamma_{t u r n}
$$

with $X \in\left\{a c, m e_{1}, m e_{2}, m e_{3}\right\}$ and $\gamma_{t u r n}=0.03 h^{-1}$ the uniform turnover rate.

- DNA replication occurs every $T=13.5 \mathrm{~h}$. During this periodic event, the state of each histone can be lost with a probability $1 / 2$ and replaced by a ' $u$ ' state.

\section{Relation between the nucleation and spreading rates}

In the epigenetic model (see above), a PRC2 complex bound to locus $i$ has a local (nucleation) activity with rates $k_{m e_{x}}$ and may have, if $i$ is trimethylated, a long-range activity on any locus $j$ with rates $\epsilon_{m e_{x}} /(j-i)^{\lambda}$ (Eq.1). Actually, this last term represents the allosterically boosted activity of PRC2 $\left(F \times k_{m e_{x}}\right)$ times the probability $P_{3 D}(i, j)$ for PRC2 in $i$ to contact $j$ in 3D: $\epsilon_{m e_{x}} /(j-i)^{\lambda} \equiv$ $F k_{m e_{x}} P_{3 D}(i, j)$ with $F$ the fold-change of PRC2 activity due to allostery. Simple polymeric arguments lead to $P_{3 D}(i, j) \approx \sqrt{6 / \pi}\left(a / d_{i j}\right)^{3}$ with $a$ the typical 3D 'capture distance' of PRC2 and $d_{i j} \approx d_{0}(j-i)^{\lambda / 3}$ the average distance between loci $i$ and $j$. Therefore $R \equiv \epsilon_{m e_{x}} / k_{m e_{x}}=$ $F \sqrt{6 / \pi}\left(a / d_{0}\right)^{3}$ is independent of $x$. Assuming that $a \sim 10 \mathrm{~nm}$ the typical size of PRC2 complex (94) and $d_{i j} \sim 100 \mathrm{~nm}$ for 10-kbp (|j-i| 100histones) genomic distance (95) (ie, $d_{0} \sim 22 \mathrm{~nm}$ ), $F \sim 8 \times R$.

\section{Simulations of the model}

For given parameters (Table 1) of the epigenetic model and for given profiles for Suz12, p300 and UTX (Fig.1B), the corresponding stochastic dynamics (Fig.S3) of the system was simulated using a home-made Gillespie algorithm (96) implemented in Python 3.6 that is available at https://github.com/physical-biology-of-chromatin/PcG-mESC. For data in Figures 2, 3, 4 and 5, 32 independent trajectories per parameter set were simulated over 25 cell cycles to ensure the system has reached a periodic steady-state. Predicted average profiles of $\mathrm{H} 3 \mathrm{~K} 27 \mathrm{ac} / \mathrm{u} / \mathrm{me} 1 / \mathrm{me} 2 / \mathrm{me} 2$ correspond to the probability for a given locus to carry a given mark in an asynchronous cell population (i.e. averaged over time and trajectories) at steady-state. For data in Fig.6, 500 independent trajectories were simulated over 100h. In Fig.6A (cell cycle dynamics), average proportions along the cell cycle for each mark are given for a synchronized cell population (i.e. averaged over trajectories and over various cell cycles) that has reached a periodic steady-state. In Figs. 6C-E, average values correspond to a population of asynchronized 
cells (i.e., at a given absolute time, different trajectories may correspond to different relative times along the cell cycle) for which we tracked the replacement of histones by turnover or dilution from a given absolute time $(\mathrm{t}=50 \mathrm{~h})$. In addition, for Figs. $6 \mathrm{G}-\mathrm{H}$, the asynchronized population was first evolved in a $\mathrm{EZH} 2 \mathrm{i}$-like situation $\left(\epsilon_{m e_{x}}=0\right)$ until steady-state before being switched to a WT-like situation at a given absolute time $(\mathrm{t}=50 \mathrm{~h})$.

\section{Profiles of HMEs used in the simulations}

To simulate the average epigenetic landscape around PcG-target, active or bivalent genes in $2 \mathrm{i}$ condition (Fig.2-4), we directly used the average normalized Chip-Seq profiles of p300, UTX and SUZ12 around TSS for each gene category (Fig.S5), as described above. Note that SUZ12 profiles have been well measured in $2 \mathrm{i}$ condition but p300 and UTX are taken from serum condition experiments (Table 2). However, we assumed that p300 and UTX profiles are also valid for the $2 \mathrm{i}$ condition for all gene categories.

For predictions around single genes (Fig.4C), the noisy normalized Chip-Seq profiles (50 bpbinning) were smoothed out with a moving average over a $300 \mathrm{bp}$-long window.

To investigate the interplay between the recruitment strengths of p300/UTX and PRC2 (Fig.5), we first fitted the average HME profiles (Fig.S5) by Gaussian-like functions: $\psi_{m} \exp \left[-\left(i-i_{0}\right)^{2} /\right.$ $\left.\left(2 \sigma_{0}^{2}\right)\right]+\psi_{b}$ with $\psi_{b}$ the background level, $\psi_{m}$ the height of the binding peak from background, $i_{0}$ the position of the peak and $\sigma_{0}$ the typical width of the peak. Then, we predicted the average H3K27 proportions for hypothetical genes characterized by a Suz12/PRC2 profile $\psi_{\text {Suz } 12}(i)=\alpha \psi_{m, \text { Suz } 12}(P c G) \exp \left[-\left(i-i_{0, \text { Suz } 12}\right)^{2} /\left(2 \sigma_{0, \text { Suz } 12}^{2}\right)\right]+\psi_{b, \text { Suz } 12}, \quad$ a $\quad$ p300 profile $\psi_{p 300}(i)=\beta \psi_{m, p 300}($ Act. $) \exp \left[-\left(i-i_{0, p 300}\right)^{2} /\left(2 \sigma_{0, p 300}^{2}\right)\right]+\psi_{b, p 300}$ and a UTX profile $\psi_{U T X}(i)=\beta \psi_{m, U T X}($ Act. $) \exp \left[-\left(i-i_{0, U T X}\right)^{2} /\left(2 \sigma_{0, U T X}^{2}\right)\right]+\psi_{b, U T X}$ where $\psi_{m, S u z 12}(P c G)$ is the amplitude measured around PcG-target genes and $\psi_{m, p 300 / U T X}($ Act.) around active genes, $\alpha$ and $\beta$ are two multiplicative factors allowing to vary the amplitudes of the HME profiles around TSS. Here, for simplicity, we assumed that p300 and UTX evolved with the same factor $\beta$. For example, WT PcG-target gene in $2 \mathrm{i}$ condition corresponds to $(\alpha ; \beta) \approx(1 ; 0.3)$ and active genes to $(\alpha ; \beta) \approx$ $(0 ; 1)$.

\section{Parameter inference}

To fix the remaining free parameters of the model, we develop a multi-step inference strategy based on the different perturbation experiments (EZH1/2 DKO, EZH2 KO, Wild Type and UTX inhibited) from $(29,32)$. To be self-consistent, the fitting was carried out exclusively with the average profiles obtained for PcG-target genes (see above). 


\section{Acetylation rate}

In EZH1/2 DKO cells, the epigenetic model becomes a two-state model between the 'ac' and ' $u$ ' states since methylation is not possible. In this simple case, the probability of being acetylated at position $i$ is given by

$P_{a c}(i)=k_{a c} \psi_{p 300}(i) /\left[k_{a c} \psi_{p 300}(i)+\gamma_{a c}+\gamma_{t u r n}+\log (2) / T\right]$

The p300 ChipSeq density $\psi_{p 300}$ for EZH1/2 DKO cells was unavailable, so we used the wild type p300 occupancy with a correction factor. We first fitted the WT profile by a Gaussian-like function (full line in Fig.2B), $\psi_{p 300}^{W T}(i)=\psi_{m} \exp \left[-\left(i-i_{0}\right)^{2} /\left(2 \sigma_{0}^{2}\right)\right]+\psi_{b}$, with $\psi_{b}=0.1196$ the background, non-specific binding level of p300, $\psi_{m}=0.1666$ the specific maximal increase of binding at the peak, $i_{0}=-0.5438$ the position of the peak and $\sigma_{0}=5.1875$ the typical width of the peak. Assuming a uniform difference in p300 occupancy between WT and DKO situations, we model the DKO p300 profile as $\psi_{p 300}^{D K O}(i)=\psi_{m} \exp \left[-\left(i-i_{0}\right)^{2} /\left(2 \sigma_{0}^{2}\right)\right]+\alpha \psi_{b}$ with $\alpha<1$ the correction factor. Using this profile in Eq. 6 and minimizing a chi-squared score between model predictions and experiments (Fig.2C), we can infer $k_{a c}=1.03 h^{-1}$ and $\alpha=0.6$ (see also Fig.S10).

\section{(De)methylation rates}

Inference of methylation-related parameters follows an iterative multi-steps strategy as described in the main text.

1. Ratios $r_{13} \equiv k_{m e 1} / k_{m e 3}$ and $r_{23} \equiv k_{m e 2} / k_{m e 3}$ are fixed to arbitrary values.

2. The absolute nucleation rate $k_{m e 3}$ is initialized to a random value.

3. In our model, EZH2 KO cells correspond to an epigenetic system without spreading $\left(\epsilon_{m e_{x}}=0\right)$. For various values of $\gamma_{m e}$, we simulated this situation using the SUZ12 Chipseq density measured in EZH2 KO by Lavarone et al (29). We could not find the UTX/p300 occupancy of EZH2KO cells in literature, so wild type UTX/p300 occupancy was used. At this point, we do not see any indication that knockout of EZH2 will significantly alter the presence of UTX/p300 occupancy for PcG genes. As normalized Chip-seq densities are more pertinent in terms of relative comparison, we fixed the value of $\gamma_{m e}$ that is qualitatively consistent with the methylation valency observed around the promoter of PcG-target genes in $\mathrm{EZH} 2 \mathrm{KO}$ cells, i.e. the profile of me2> profile of me3> profile of me1 at TSS (Fig.2F-H).

4. Using wild-type cells data, we then fixed the spreading rates $\epsilon_{m e_{x}}$, or more exactly the ratio $R=\epsilon_{m e_{x}} / k_{m e_{x}}$. Again, estimation of $R$ was based on capturing the methylation valency at PcG-target genes rather than absolute Chip-Seq density, such that H3K27me3 is prevalent in the large regions around TSS's and eventually overtaken by H3K27me2 at $\sim 5( \pm 0.5) \mathrm{kb}$ from TSS (Fig.2J-K).

5. At this point, all parameters have been fixed $\left(k_{m e 1 / m e 2 / m e 3}\right)$ or inferred $\left(\gamma_{m e}, R\right)$. We used MINUTE Chip experiments for UTX inhibited cells to validate these parameters. The corresponding H3K27me3 experimental profile has been calibrated such that it quantitatively corresponds to the probability that H3K27 is trimethylated (32) (see above) and can therefore be directly compared to model predictions. We simulated these cells with $\gamma_{m e}=0$ and neglecting the acetylation pathway considering that UTX is a stimulant 
for p300 recruitment (46). By keeping $R$ to the inferred value in step 4 and $r_{13}$ and $r_{23}$ to the imposed values in step 1 , we corrected the $k_{m e 3}$ value to minimize a chi-squared distance between predictions and experiments.

6. Steps 3 to 5 are repeated until convergence (Fig.S1) or failure of the fitting process when steps 3 \& 4 cannot capture the experimental methylation valencies.

7. Steps 2 to 6 are repeated for different initial values of $k_{m e 3}$ (Fig.S1). We found that, in absence of failure, the strategy always converged to the same final parameter values.

8. Steps 1 to 7 are repeated for different values of $r_{13}$ and $r_{23}$. We limited the scanning of these ratios to integer values between 1 and 9 with setting $r_{13} \geq r_{23}$ as suggested by the in vitro experiment (50).

\section{Correlations of the local epigenomic state}

In Fig. 3B, the correlation $C_{i, j}(x, y)$ between the state $x$ of locus $i$ and the state $y$ of locus $j(x, y \in$ $\left.\left\{a c, u, m e_{1}, m e_{2}, m e_{3}\right\}\right)$ is given by the Pearson correlation correlation between the random variables $\delta_{i}(x)$ and $\delta_{j}(y)$ where $\delta_{i}(x)=1$ if the H3K27 state of locus $i$ is $x(=0$ otherwise) in the current simulated configuration:

$$
C_{i, j}(x, y)=\left(<\delta_{i}(x) \delta_{j}(y)>-<\delta_{i}(x)><\delta_{j}(y)>\right) / \sqrt{<\delta_{i}(x)><\delta_{j}(y)>\left(1-<\delta_{i}(x)>\right)\left(1-<\delta_{j}(y)>\right)}
$$

with $<.>$ the time and population average of the given random variable.

\section{Data extraction from SILAC experiments}

Model predictions on the dynamics of H3K27 modifications (Fig.6) are compared to experimental data obtained using the SILAC and mass spectrometry technologies that measure the global, genome-wide proportions of a given modification in different pools of histones $(59,60)$.

Data in Fig.6B on the cell cycle dynamics of the different marks in all histones were obtained by averaging the proportions a given mark in the pools of old (light medium) and new (heavy medium) histones at different times after the release into $S$ phase (which corresponds also to the moment of medium change) extracted from Fig.3E and Fig.S4C of (60). Here, we arguably assumed that after replication and during one cell cycle, the pools of old and new are of similar sizes, as also done in Fig.3A of (60).

Data in Fig.6D,F on the dynamics of the marks in new and old histones were obtained from Fig.1D-F and Fig.S1A of (59). The dynamics in the new histone pool were directly extracted from Fig.1F, left (Generation 3). The dynamics in the old histone pool were computed as the weighted average of the Generation 1 (extracted from Fig.1E, left) and Generation 2 (extracted from Fig.1D, left) data. The weight for each generation at a given time was taken proportional to the percentage of Generation 1 or 2 histones among all the histones (extracted from Fig.S1A).

Data in Fig.6G (bottom) on the dynamics of the marks in all histones after the release of the EZH2 inhibitor were directly extracted from Fig.S3C of (59). 
In Fig.6A,B, to correct for differences in cell cycle lengths between simulations that are made on mESC and experiments made on HeLa cells, time after replication is normalized by the corresponding cell cycle length ( $T=13.5 \mathrm{~h}$ for mESC simulations, $T=24 \mathrm{~h}$ for HeLa cells).

In Fig.6C-H, to correct for differences in global histone turnover rates between simulations and experiments, time is normalized by the corresponding effective histone decay time $t_{e}$ that captures the combined effect of direct histone turnover and of dilution after replication in a population of unsynchronized cells. $t_{e}$ is equal to the characteristic time of decay of the proportion of 'old' histones among all histones and can be obtained by fitted the corresponding curves by $\exp \left(-t / t_{e}\right)$. For simulations, we estimated $t_{e}=12.7 \mathrm{~h}$. For experiments, we obtained $t_{e}=28.6 \mathrm{~h}$ by fitting the time-evolution of the sum of the proportions of Generation 1 and of Generation 2 extracted from Fig.S1A of (59).

\section{Acknowledgements}

We acknowledge fruitful discussions with Cédric Vaillant, Amith Zafal Abdulla, Guillermo Orsi, Sonja Prohaska and members of the SYMER consortium. We thank Emma Chory and Gerald Crabtree for sharing their Chip-Seq normalization pipeline and the list of repressed, bivalent and active genes inferred in (25), as well as Simon Elsaseer for useful insights on the MINUTE Chip method.

\section{Funding}

The research leading to these results was supported by the University Grenoble-Alpes via the SYMER program (which is funded by the French National Research Agency under the "Investissements d'Avenir" program ANR-15-IDEX-02). DJ acknowledges additional funding from the French National Research Agency [ANR-18-CE12-0006-03, ANR-18-CE45-0022-01]. EF acknowledges funding from ITMO Cancer in the framework of the French Plan Cancer (Systems Biology program).

\section{Author's contributions}

K.N.: Conceptualization, Bioinformatics, Modeling, Software, Investigation, Data analysis, Writing. E.F.: Conceptualization, Modeling, Data analysis, Supervision, Funding acquisition. D.J: Conceptualization, Modeling, Investigation, Data analysis, Supervision, Funding acquisition, Writing.

\section{Conflict of interests}

The authors declare that they have no conflict of interest. 


\section{References}

1. David Allis C. Epigenetics. CSHL Press; 2007. 502 p.

2. Jenuwein T, Allis CD. Translating the histone code. Science. 2001 Aug 10;293(5532):1074-80.

3. Pujadas E, Feinberg AP. Regulated noise in the epigenetic landscape of development and disease. Cell. 2012 Mar 16;148(6):1123-31.

4. Beisel C, Paro R. Silencing chromatin: comparing modes and mechanisms. Nat Rev Genet. 2011 Feb;12(2):123-35.

5. Ragunathan K, Jih G, Moazed D. Epigenetics. Epigenetic inheritance uncoupled from sequencespecific recruitment. Science. 2015 Apr 3;348(6230):1258699.

6. Oksuz O, Narendra V, Lee C-H, Descostes N, LeRoy G, Raviram R, et al. Capturing the Onset of PRC2-Mediated Repressive Domain Formation. Mol Cell. 2018 Jun 21;70(6):1149-62.e5.

7. Zhang T, Cooper S, Brockdorff N. The interplay of histone modifications - writers that read. EMBO Rep. 2015 Nov; 16(11):1467-81.

8. Dodd IB, Micheelsen MA, Sneppen K, Thon G. Theoretical analysis of epigenetic cell memory by nucleosome modification. Cell. 2007 May 18;129(4):813-22.

9. Jost D. Bifurcation in epigenetics: implications in development, proliferation, and diseases. Phys Rev E Stat Nonlin Soft Matter Phys. 2014 Jan;89(1):010701.

10. Dodd IB, Sneppen K. Barriers and silencers: a theoretical toolkit for control and containment of nucleosome-based epigenetic states. J Mol Biol. 2011 Dec 9;414(4):624-37.

11. David-Rus D, Mukhopadhyay S, Lebowitz JL, Sengupta AM. Inheritance of epigenetic chromatin silencing. Journal of Theoretical Biology. 2009; 258: 112-20.

12. Folguera-Blasco $\mathrm{N}$, Cuyàs $\mathrm{E}$, Menéndez JA, Alarcón $T$. Epigenetic regulation of cell fate reprogramming in aging and disease: A predictive computational model. PLOS Computational Biology. 2018; 14: e1006052.

13. Angel A, Song J, Dean C, Howard M. A Polycomb-based switch underlying quantitative epigenetic memory. Nature. 2011; 476: 105-8.

14. Berry S, Dean C, Howard M. Slow Chromatin Dynamics Allow Polycomb Target Genes to Filter Fluctuations in Transcription Factor Activity. Cell Syst. 2017 Apr 26;4(4):445-57.e8.

15. Erdel F, Greene EC. Generalized nucleation and looping model for epigenetic memory of histone modifications. Proceedings of the National Academy of Sciences. 2016; 113: E4180-9.

16. Sneppen K, Ringrose L. Theoretical analysis of Polycomb-Trithorax systems predicts that poised chromatin is bistable and not bivalent. Nat Commun. 2019 May 13;10(1):2133.

17. Reinig J, Ruge F, Howard M, Ringrose L. A theoretical model of Polycomb/Trithorax action unites stable epigenetic memory and dynamic regulation. Nat Commun. 2020 Sep 22;11(1):4782.

18. Lövkvist $\mathrm{C}$, Howard $\mathrm{M}$. Using computational modelling to reveal mechanisms of epigenetic Polycomb control. Biochem Soc Trans. 2021 Feb 26;49(1):71-7.

19. Ringrose L, Howard M. Dissecting chromatin-mediated gene regulation and epigenetic memory through mathematical modelling. Current Opinion in Systems Biology. 2017; 3: 7-14.

20. Obersriebnig MJ, Pallesen EMH, Sneppen K, Trusina A, Thon G. Nucleation and spreading of a heterochromatic domain in fission yeast. Nat Commun. 2016 May 11;7:11518.

21. Nickels JF, Edwards AK, Charlton SJ, Mortensen AM, Hougaard SCL, Trusina A, et al. Establishment of heterochromatin in domain-size-dependent bursts. Proc Natl Acad Sci U S A. 2021 Apr 13;118(15).

22. Angel A, Song J, Yang H, Questa JI, Dean C, Howard M. Vernalizing cold is registered digitally at FLC. Proc Natl Acad Sci U S A. 2015 Mar 31;112(13):4146-51.

23. Yang $\mathrm{H}$, Howard $\mathrm{M}$, Dean $\mathrm{C}$. Physical coupling of activation and derepression activities to maintain an active transcriptional state at FLC. Proc Natl Acad Sci U S A. 2016 Aug 16;113(33):9369-74.

24. Nagaraj VH, Mukhopadhyay S, Dayarian A, Sengupta AM. Breaking an epigenetic chromatin switch: curious features of hysteresis in Saccharomyces cerevisiae telomeric silencing. PLoS One. 2014 Dec 23;9(12):e113516.

25. Chory EJ, Calarco JP, Hathaway NA, Bell O, Neel DS, Crabtree GR. Nucleosome Turnover Regulates Histone Methylation Patterns over the Genome. Mol Cell. 2019 Jan 3;73(1):61-72.e3.

26. Schuettengruber B, Bourbon H-M, Di Croce L, Cavalli G. Genome Regulation by Polycomb and Trithorax: 70 Years and Counting. Cell. 2017 Sep 21;171(1):34-57. 
27. Piunti A, Shilatifard A. The roles of Polycomb repressive complexes in mammalian development and cancer. Nat Rev Mol Cell Biol. 2021 May;22(5):326-45.

28. Juan AH, Wang S, Ko KD, Zare H, Tsai P-F, Feng X, et al. Roles of H3K27me2 and H3K27me3 Examined during Fate Specification of Embryonic Stem Cells. Cell Rep. 2017 Jan 3;18(1):297.

29. Lavarone E, Barbieri CM, Pasini D. Dissecting the role of H3K27 acetylation and methylation in PRC2 mediated control of cellular identity. Nat Commun. 2019 Apr 11;10(1):1679.

30. Laugesen A, Højfeldt JW, Helin K. Molecular Mechanisms Directing PRC2 Recruitment and H3K27 Methylation. Mol Cell. 2019 Apr 4;74(1):8-18.

31. Højfeldt JW, Laugesen A, Willumsen BM, Damhofer H, Hedehus L, Tvardovskiy A, et al. Accurate H3K27 methylation can be established de novo by SUZ12-directed PRC2. Nat Struct Mol Biol. 2018 Mar;25(3):225-32.

32. Kumar B, Elsässer SJ. Quantitative Multiplexed ChIP Reveals Global Alterations that Shape Promoter Bivalency in Ground State Embryonic Stem Cells. Cell Rep. 2019 Sep 17;28(12):3274-84.e5.

33. Aljazi MB, Gao Y, Wu Y, Mias GI, He J. Cell Signaling Coordinates Global PRC2 Recruitment and Developmental Gene Expression in Murine Embryonic Stem Cells. iScience. 2020 Nov 20;23(11):101646.

34. Mikkelsen TS, Ku M, Jaffe DB, Issac B, Lieberman E, Giannoukos G, et al. Genome-wide maps of chromatin state in pluripotent and lineage-committed cells. Nature. 2007; 448: 553-60.

35. Moussa HF, Bsteh D, Yelagandula R, Pribitzer C, Stecher K, Bartalska K, et al. Canonical PRC1 controls sequence-independent propagation of Polycomb-mediated gene silencing. Nat Commun. 2019 Apr 29;10(1):1931.

36. Blackledge NP, Fursova NA, Kelley JR, Huseyin MK, Feldmann A, Klose RJ. PRC1 Catalytic Activity Is Central to Polycomb System Function. Mol Cell. 2020 Feb 20;77(4):857-74.e9.

37. Tamburri S, Lavarone E, Fernández-Pérez D, Conway E, Zanotti M, Manganaro D, et al. Histone H2AK119 Mono-Ubiquitination Is Essential for Polycomb-Mediated Transcriptional Repression. Mol Cell. 2020 Feb 20;77(4):840-56.e5.

38. Blackledge NP, Farcas AM, Kondo T, King HW, McGouran JF, Hanssen LLP, et al. Variant PRC1 complex-dependent $\mathrm{H} 2 \mathrm{~A}$ ubiquitylation drives PRC2 recruitment and polycomb domain formation. Cell. 2014 Jun 5;157(6):1445-59.

39. Kalb R, Latwiel S, Baymaz HI, Jansen PWTC, Müller CW, Vermeulen M, et al. Histone H2A monoubiquitination promotes histone $\mathrm{H} 3$ methylation in Polycomb repression. Nat Struct Mol Biol. 2014 Jun;21(6):569-71.

40. Cooper S, Dienstbier M, Hassan R, Schermelleh L, Sharif J, Blackledge NP, et al. Targeting polycomb to pericentric heterochromatin in embryonic stem cells reveals a role for H2AK119u1 in PRC2 recruitment. Cell Rep. 2014 Jun 12;7(5):1456-70.

41. Pasini D, Cloos PAC, Walfridsson J, Olsson L, Bukowski J-P, Johansen JV, et al. JARID2 regulates binding of the Polycomb repressive complex 2 to target genes in ES cells. Nature. 2010 Mar 11;464(7286):306-10.

42. Healy E, Mucha M, Glancy E, Fitzpatrick DJ, Conway E, Neikes HK, et al. PRC2.1 and PRC2.2 Synergize to Coordinate H3K27 Trimethylation. Mol Cell. 2019 Nov 7;76(3):437-52.e6.

43. Petracovici A, Bonasio R. Distinct PRC2 subunits regulate maintenance and establishment of Polycomb repression during differentiation. Mol Cell. 2021 Jun 17;81(12):2625-39.e5.

44. Lee C-H, Holder M, Grau D, Saldaña-Meyer R, Yu J-R, Ganai RA, et al. Distinct stimulatory mechanisms regulate the catalytic activity of polycomb repressive complex 2. Mol Cell. 2018 May 3;70(3):435-48.e5.

45. Isono K, Endo TA, Ku M, Yamada D, Suzuki R, Sharif J, et al. SAM domain polymerization links subnuclear clustering of PRC1 to gene silencing. Dev Cell. 2013 Sep 30;26(6):565-77.

46. Wang S-P, Tang Z, Chen C-W, Shimada M, Koche RP, Wang L-H, et al. A UTX-MLL4-p300 Transcriptional Regulatory Network Coordinately Shapes Active Enhancer Landscapes for Eliciting Transcription. Mol Cell. 2017 Jul 20;67(2):308-21.e6.

47. Agger K, Cloos PAC, Christensen J, Pasini D, Rose S, Rappsilber J, et al. UTX and JMJD3 are histone H3K27 demethylases involved in HOX gene regulation and development. Nature. 2007 Oct 11;449(7163):731-4.

48. Chronis C, Fiziev P, Papp B, Butz S, Bonora G, Sabri S, et al. Cooperative binding of transcription factors orchestrates reprogramming. Cell. 2017 Jan 26;168(3):442-59.e20.

49. Martire S, Nguyen J, Sundaresan A, Banaszynski LA. Differential contribution of p300 and CBP to 
regulatory element acetylation in mESCs. BMC Mol Cell Biol. 2020 Jul 20;21(1):55.

50. McCabe MT, Graves AP, Ganji G, Diaz E, Halsey WS, Jiang Y, et al. Mutation of A677 in histone methyltransferase EZH2 in human B-cell lymphoma promotes hypertrimethylation of histone $\mathrm{H} 3$ on lysine 27 (H3K27). Proc Natl Acad Sci U S A. 2012 Feb 21;109(8):2989-94.

51. Ferrari KJ, Scelfo A, Jammula S, Cuomo A, Barozzi I, Stützer A, et al. Polycomb-dependent H3K27me1 and H3K27me2 regulate active transcription and enhancer fidelity. Mol Cell. 2014 Jan 9;53(1):49-62.

52. Zee BM, Britton L-MP, Wolle D, Haberman DM, Garcia BA. Origins and formation of histone methylation across the human cell cycle. Mol Cell Biol. 2012 Jul;32(13):2503-14.

53. Kuzmichev A, Nishioka K, Erdjument-Bromage H, Tempst P, Reinberg D. Histone methyltransferase activity associated with a human multiprotein complex containing the Enhancer of Zeste protein. Genes Dev. 2002 Nov 15;16(22):2893-905.

54. Raisner R, Kharbanda S, Jin L, Jeng E, Chan E, Merchant M, et al. Enhancer Activity Requires CBP/P300 Bromodomain-Dependent Histone H3K27 Acetylation. Cell Rep. 2018 Aug 14;24(7):17229.

55. Weinert BT, Narita T, Satpathy S, Srinivasan B, Hansen BK, Schölz C, et al. Time-Resolved Analysis Reveals Rapid Dynamics and Broad Scope of the CBP/p300 Acetylome. Cell. 2018 Jun 28;174(1):231-44.e12.

56. Zheng Y, Thomas PM, Kelleher NL. Measurement of acetylation turnover at distinct lysines in human histones identifies long-lived acetylation sites. Nat Commun. 2013;4:2203.

57. Banaszynski LA, Wen D, Dewell S, Whitcomb SJ, Lin M, Diaz N, et al. Hira-dependent histone H3.3 deposition facilitates PRC2 recruitment at developmental loci in ES cells. Cell. 2013 Sep 26;155(1):107-20.

58. Deaton AM, Gómez-Rodríguez M, Mieczkowski J, Tolstorukov MY, Kundu S, Sadreyev RI, et al. Enhancer regions show high histone $\mathrm{H} 3.3$ turnover that changes during differentiation. Elife. 2016 Jun $15 ; 5$.

59. Alabert C, Loos C, Voelker-Albert M, Graziano S, Forné I, Reveron-Gomez N, et al. Domain Model Explains Propagation Dynamics and Stability of Histone H3K27 and H3K36 Methylation Landscapes. Cell Rep. 2020 Jan 28;30(4):1223-34.e8.

60. Alabert C, Barth TK, Reverón-Gómez N, Sidoli S, Schmidt A, Jensen ON, et al. Two distinct modes for propagation of histone PTMs across the cell cycle. Genes Dev. 2015 Mar 15;29(6):585-90.

61. Yu C, Gan H, Serra-Cardona A, Zhang L, Gan S, Sharma S, et al. A mechanism for preventing asymmetric histone segregation onto replicating DNA strands. Science. 2018 Sep 28;361(6409):1386-9.

62. Petryk N, Dalby M, Wenger A, Stromme CB, Strandsby A, Andersson R, et al. MCM2 promotes symmetric inheritance of modified histones during DNA replication. Science. 2018 Sep 28;361(6409):1389-92.

63. Waisman A, Sevlever F, Elías Costa M, Cosentino MS, Miriuka SG, Ventura AC, et al. Cell cycle dynamics of mouse embryonic stem cells in the ground state and during transition to formative pluripotency. Sci Rep. 2019 May 29;9(1):8051.

64. Weinert BT, Satpathy S, Hansen BK, Lyon D, Jensen LJ, Choudhary C. Accurate Quantification of Site-specific Acetylation Stoichiometry Reveals the Impact of Sirtuin Deacetylase CobB on the E. coli Acetylome. Mol Cell Proteomics. 2017 Mar 2;16(5):759-69.

65. Evertts AG, Zee BM, Dimaggio PA, Gonzales-Cope M, Coller HA, Garcia BA. Quantitative dynamics of the link between cellular metabolism and histone acetylation. J Biol Chem. 2013 Apr 26;288(17):12142-51.

66. Margueron R, Justin N, Ohno K, Sharpe ML, Son J, Drury WJ 3rd, et al. Role of the polycomb protein EED in the propagation of repressive histone marks. Nature. 2009 Oct 8;461(7265):762-7.

67. Grimaud C, Nègre N, Cavalli G. From genetics to epigenetics: the tale of Polycomb group and trithorax group genes. Chromosome Res. 2006;14(4):363-75.

68. Jain SU, Rashoff AQ, Krabbenhoft SD, Hoelper D, Do TJ, Gibson TJ, et al. H3 K27M and EZHIP Impede H3K27-Methylation Spreading by Inhibiting Allosterically Stimulated PRC2. Mol Cell. 2020 Nov 19;80(4):726-35.e7.

69. Ghotbi E, Ye P, Ervin T, Kum A, Benes J, Jones RS. Polycomb-group recruitment to a target gene is the default state that is inhibited by a transcriptional activator. Sci Adv. $2021 \mathrm{Jul} ; 7(29)$.

70. Zerihun $\mathrm{MB}$, Vaillant $\mathrm{C}$, Jost $\mathrm{D}$. Effect of replication on epigenetic memory and consequences on gene 
transcription. Phys Biol. 2015 Apr 17;12(2):026007.

71. Ziane R, Camasses A, Radman-Livaja M. Mechanics of DNA Replication and Transcription Guide the Asymmetric Distribution of RNAPol2 and Nucleosomes on Replicated Daughter Genomes. bioRxiv, available from: http://dx.doi.org/10.1101/553669

72. Laprell F, Finkl K, Müller J. Propagation of Polycomb-repressed chromatin requires sequence-specific recruitment to DNA. Science. 2017 Apr 7;356(6333):85-8.

73. Torné J, Ray-Gallet D, Boyarchuk E, Garnier M, Le Baccon P, Coulon A, et al. Two HIRA-dependent pathways mediate H3.3 de novo deposition and recycling during transcription. Nat Struct Mol Biol. 2020 Nov;27(11):1057-68.

74. Deal RB, Henikoff JG, Henikoff S. Genome-wide kinetics of nucleosome turnover determined by metabolic labeling of histones. Science. 2010 May 28;328(5982):1161-4.

75. Streubel G, Watson A, Jammula SG, Scelfo A, Fitzpatrick DJ, Oliviero G, et al. The H3K36me2 Methyltransferase Nsd1 Demarcates PRC2-Mediated H3K27me2 and H3K27me3 Domains in Embryonic Stem Cells. Molecular Cell. 2018 ;70: 371-9.e5.

76. Cheutin T, Cavalli G. Progressive polycomb assembly on H3K27me3 compartments generates polycomb bodies with developmentally regulated motion. PLoS Genet. 2012 Jan;8(1):e1002465.

77. Pirrotta V, Li H-B. A view of nuclear Polycomb bodies. Curr Opin Genet Dev. 2012 Apr;22(2):101-9.

78. Plys AJ, Davis CP, Kim J, Rizki G, Keenen MM, Marr SK, et al. Phase separation of Polycombrepressive complex 1 is governed by a charged disordered region of CBX2. Genes Dev. 2019 Jul 1;33(13-14):799-813.

79. Francis NJ, Kingston RE, Woodcock CL. Chromatin compaction by a polycomb group protein complex. Science. 2004 Nov 26;306(5701):1574-7.

80. Eagen KP, Aiden EL, Kornberg RD. Polycomb-mediated chromatin loops revealed by a subkilobaseresolution chromatin interaction map. Proc Natl Acad Sci U S A. 2017 Aug 15;114(33):8764-9.

81. Seif E, Kang JJ, Sasseville C, Senkovich O, Kaltashov A, Boulier EL, et al. Phase separation by the polyhomeotic sterile alpha motif compartmentalizes Polycomb Group proteins and enhances their activity. Nat Commun. 2020 Nov 5;11(1):5609.

82. Haddad N, Jost D, Vaillant C. Perspectives: using polymer modeling to understand the formation and function of nuclear compartments. Chromosome Res. 2017 Mar;25(1):35-50.

83. Jost D, Vaillant C, Meister P. Coupling 1D modifications and 3D nuclear organization: data, models and function. Curr Opin Cell Biol. 2017 Feb;44:20-7.

84. Jost $D$, Vaillant $C$. Epigenomics in $3 D$ : importance of long-range spreading and specific interactions in epigenomic maintenance. Nucleic Acids Res. 2018 Mar 16;46(5):2252-64.

85. Michieletto D, Orlandini E, Marenduzzo D. A Polymer Model with Epigenetic Recolouring Reveals a Pathway for the de novo Establishment and 3D organisation of Chromatin Domains. Phys Rev X. 2016; 6: 041047.

86. Murawska M, Greenstein RA, Schauer T, Olsen KCF, $\mathrm{Ng} \mathrm{H}$, Ladurner AG, et al. The histone chaperone FACT facilitates heterochromatin spreading through regulation of histone turnover and H3K9 methylation states. bioRxiv. Available from: http://dx.doi.org/10.1101/2021.06.30.450523

87. Afgan E, Baker D, Batut B, van den Beek M, Bouvier D, Cech M, et al. The Galaxy platform for accessible, reproducible and collaborative biomedical analyses: 2018 update. Nucleic Acids Res. 2018 Jul 2;46(W1):W537-44.

88. Langmead B, Salzberg SL. Fast gapped-read alignment with Bowtie 2. Nat Methods. 2012 Mar 4;9(4):357-9.

89. Li H, Handsaker B, Wysoker A, Fennell T, Ruan J, Homer N, et al. The Sequence Alignment/Map format and SAMtools. Bioinformatics. 2009 Aug 15;25(16):2078-9.

90. Orlando DA, Chen MW, Brown VE, Solanki S, Choi YJ, Olson ER, et al. Quantitative ChIP-Seq normalization reveals global modulation of the epigenome. Cell Rep. 2014 Nov 6;9(3):1163-70.

91. Ramírez F, Ryan DP, Grüning B, Bhardwaj V, Kilpert F, Richter AS, et al. deepTools2: a next generation web server for deep-sequencing data analysis. Nucleic Acids Res. 2016 Jul 8;44(W1):W160-5.

92. Mirny LA. The fractal globule as a model of chromatin architecture in the cell. Chromosome Res. 2011 Jan;19(1):37-51.

93. Bonev B, Mendelson Cohen N, Szabo Q, Fritsch L, Papadopoulos GL, Lubling Y, et al. Multiscale 3D Genome Rewiring during Mouse Neural Development. Cell. 2017 Oct 19;171(3):557-72.e24.

94. Justin N, Zhang Y, Tarricone C, Martin SR, Chen S, Underwood E, et al. Structural basis of oncogenic 
bioRxiv preprint doi: https://doi.org/10.1101/2021.09.30 462529; this version posted December 15, 2021. The copyright holder for this preprint (which was not certified by peer review) is the author/funder, who has granted bioRxiv a license to display the preprint in perpetuity. It is made available under aCC-BY 4.0 International license.

histone H3K27M inhibition of human polycomb repressive complex 2. Nat Commun. 2016 Apr 28;7:11316.

95. Kimura H, Shimooka Y, Nishikawa J-I, Miura O, Sugiyama S, Yamada S, et al. The genome folding mechanism in yeast. J Biochem. 2013 Aug;154(2):137-47.

96. Gillespie DT. Exact stochastic simulation of coupled chemical reactions. J Phys Chem. 1977 Dec;81(25):2340-61. 